\title{
Scaling-Up and Model Inversion Methods with Narrowband Optical Indices for Chlorophyll Content Estimation in Closed Forest Canopies with Hyperspectral Data
}

\author{
Pablo J. Zarco-Tejada, John R. Miller, Thomas L. Noland, Gina H. Mohammed, and Paul H. Sampson
}

\begin{abstract}
Radiative transfer theory and modeling assumptions were applied at laboratory and field scales in order to study the link between leaf reflectance and transmittance and canopy hyperspectral data for chlorophyll content estimation. This study was focused on 12 sites of Acer saccharum M. (sugar maple) in the Algoma Region, Canada, where field measurements, laboratory-simulation experiments, and hyperspectral compact airborne spectrographic imager (CASI) imagery of 72 channels in the visible and near-infrared region and up to 1-m spatial resolution data were acquired in the 1997, 1998, and 1999 campaigns. A different set of 14 sites of the same species were used in 2000 for validation of methodologies. Infinite reflectance and canopy reflectance models were used to link leaf to canopy levels through radiative transfer simulation. The closed and dense $(L A I>4)$ forest canopies of Acer saccharum M. used for this study, and the high spatial resolution reflectance data targeting crowns, allowed the use of optically thick simulation formulae and turbid-medium SAILH and MCRM canopy reflectance models for chlorophyll content estimation by scaling-up and by numerical model inversion approaches through coupling to the PROSPECT leaf radiative transfer model. Study of the merit function in the numerical inversion showed that red edge optical indices used in the minimizing function such as $R_{750} / R_{710}$ perform better than when all single spectral reflectance channels from hyperspectral airborne CASI data are used, and in addition, the effect of shadows and LAI variation are minimized. Estimates of leaf pigment by hyperspectral remote sensing of closed forest canopies were shown to be feasible with root mean square errors (RMSE's) ranging from 3 to $5.5 \mu \mathrm{g} / \mathrm{cm}^{2}$. Pigment estimation by model inversion as described in this paper using these red edge in-
\end{abstract}

Manuscript received September 2, 2000; revised February 6, 2001. This work was supported in part by the Centre for Research in Earth and Space Technology (CRESTech), the Ontario Ministry of Natural Resources, the Canadian Forestry Service, the Ministry of Environment and Energy, and Geomatics for Informed Decisions (GEOIDE), part of the Canadian Networks of Centres of Excellence Programme

P. J. Zarco-Tejada was with the Centre for Research in Earth and Space Science (CRESS), York University, Toronto, ON M3J 1P3, Canada. He is now with the Department of Land, Air, and Water Resources (LAWR) Center for Spatial Technologies and Remote Sensing (CSTARS), University of California, Davis, CA 95616-8671 USA (e-mail: pzarco@ucdavis.edu).

J. R. Miller is with the Centre for Research in Earth and Space Science (CRESS), York University, Toronto, ON M3J 1P3, Canada, and also with the Department of Physics and Astronomy, York University, Toronto, ON M3J 1P3, Canada (e-mail: jrmiller@yorku.ca).

T. L. Noland is with the Ontario Forest Research Institute, Ontario Ministry of Natural Resources, Sault Ste. Marie, ON P6A 2E5, Canada.

G. H. Mohammed is with the Ontario Forest Research Institute, Ontario Ministry of Natural Resources, Sault Ste. Marie, ON P6A 2E5, Canada, and also with P\&M Technologies, Sault Ste. Marie, ON P6A 6S7, Canada.

P. H. Sampson is with the Ontario Forest Research Institute, Ontario Ministry of Natural Resources, Sault Ste. Marie, ON P6A 2E5, Canada.

Publisher Item Identifier S 0196-2892(01)05497-3. dices can in principle be readily transferred to the MERIS sensor using the $R_{750} / R_{705}$ optical index.

Index Terms-Chlorophyll, hyperspectral, leaf reflectance, optical indices, radiative transfer.

\section{INTRODUCTION}

$\mathbf{E}$ XTENSIVE research has been carried out at the leaf level in order to assess the physiological condition based on the study of the light interaction with the foliar medium. The total chlorophyll content in leaves decreases in stressed vegetation, changing the proportion of light-absorbing pigments and leading to less overall absorption with chlorophyll $a$ and $b$ $\left(\mathrm{chl}_{a+b}\right)$ being the most important plant pigments absorbing blue and red light in the 430-660 nm region, respectively [1], [2]. Differences in reflectance between healthy and stressed vegetation due to changes in pigment levels have been detected in the green peak and along the red edge (690 to $750 \mathrm{~nm})$ (e.g., [3]-[6]), allowing remote detection methods to identify vegetation stress and mapping through the influence of chlorophyll content variation [7]. Several narrowband leaf-level optical indices have been reported in the literature that might be applied to hyperspectral canopy reflectance data for $\mathrm{chl}_{a+b}$ estimation at larger scales [8], [9]. Nevertheless, most studies related to optical indices for vegetation functioning are based on measurements made at the leaf level rather than at the canopy level, where correlation between chlorophyll content and spectral reflectance can be readily observed [10]-[14]. These potentially valuable optical indices, both traditional and new developed narrowband indices derived from recent research based on reflectance and derivative spectra, are grouped into four categories, based on the spectral region and the type of parameter used [8], [9], [15]-[17].

1) Visible Ratios: SRPI $\left(R_{430} / R_{680}\right)$; NPQI $\left(R_{415}-\right.$ $\left.R_{435}\right) /\left(R_{415}+R_{435}\right)$; PRI calculated as $\left(R_{531}-\right.$ $\left.R_{570}\right) /\left(R_{531}+R_{570}\right), \quad\left(R_{550}-R_{531}\right) /\left(R_{550}+\right.$ $\left.R_{531}\right)$ and $\left(R_{570}-R_{539}\right) /\left(R_{570}+R_{539}\right) ;$ NPCI $\left(R_{680}-R_{430}\right) /\left(R_{680}+R_{430}\right)$; Carter $\left(R_{695} / R_{420}\right), \mathrm{G}$ $\left(R_{554} / R_{677}\right)$ and Lichtenthaler $\left(R_{440} / R_{690}\right)$;

2) Visible/NIR Ratios: NDVI $\left(R_{774}-R_{677}\right) /\left(R_{774}+R_{677}\right)$; $\mathrm{SR}\left(R_{774} / R_{677}\right)$; Lichtenthaler $\left(R_{800}-R_{680}\right) /\left(R_{800}+\right.$ $\left.R_{680}\right),\left(R_{440} / R_{740}\right)$; and SIPI $\left(R_{800}-R_{450}\right) /\left(R_{800}+\right.$ $\left.R_{650}\right)$ 
3) Red Edge Reflectance-Ratio Indices: Vogelmann $\left(R_{740} / R_{720}\right), \quad\left(R_{734}-R_{747}\right) /\left(R_{715}+R_{726}\right)$ $\left(R_{734}-R_{747}\right) /\left(R_{715}+R_{720}\right)$; Gitelson and Merzylak $\left(R_{750} / R_{700}\right),\left(R_{750} / R_{550}\right)$; Carter $\left(R_{695} / R_{760}\right)$; curvature index $\left(R_{675} \cdot R_{690}\right) /\left(R_{683}^{2}\right)$, and the area of the derivative under the red edge $\int_{760}^{680} D$;

4) Spectral and Derivative Red Edge Indices: the red edge inflection and chlorophyll-well wavelengths, $\lambda_{\mathbf{p}}$ and $\lambda_{\mathrm{o}}$, respectively, from red edge inverted-gaussian curve fitting [18], as well as spectral indices calculated from derivative analysis: $\left(D_{715} / D_{705}\right)$; DPR1 $\left(D \lambda_{\mathrm{p}} / D \lambda_{\mathrm{p}+12}\right), \quad$ DPR2 $\quad\left(D \lambda_{\mathrm{p}} / D \lambda_{\mathrm{p}+22}\right), \quad$ DP21 $\left(D \lambda_{\mathrm{p}} / D_{703}\right)$ and DP22 $\left(D \lambda_{\mathrm{p}} / D_{720}\right)$, where $D \lambda_{\mathrm{p}}$ is the value of the reflectance derivative at the $\lambda_{\mathrm{p}}$ spectral wavelength.

The successful application of such extensive research on leaf-level optical indices to earth observing instruments at much broader scales in order to predict canopy condition, requires the development of links between the leaf and the canopy, where photon-vegetation interactions are affected by the two different media. The estimation of pigment content at a canopy level can be performed using simple statistical relationships at a leaf level through the use of optical indices [19]-[23], using modeling methods through radiative transfer by numerical model inversion [24]-[29] and by a combination of leaf-level empirical relationships coupled with a canopy reflectance (CR) model [16], [17]. Research on the application of radiative transfer models for coupling leaf and canopy models shows promising results in the simulation of the pigment effect on leaf reflectance and in turn the effect of the geometrical arrangement of leaves on the canopy reflectance. Such developments have the potential to replace the statistically-based approaches for estimation of leaf bioindicators with quantitative model-based methods. The application of such methods in forestry canopies, where canopy structure plays an important role, the selection of the merit function used in the optimization of simulated canopy reflectance coupling a leaf and a canopy model, and the effect of leaf area index (LAI), shadows an understorey in the modeled reflectance, and therefore, in the estimated pigment content, we need continued extensive research with real airborne or satellite-level data. Throughout this paper, the term LAI represents effective leaf area index $L_{\mathrm{e}}$, as defined in [30], since $L_{\mathrm{e}}$ can be conveniently defined in terms of canopy gap fraction. Inherently, this usage ignores the effects of woody material and foliar clumping needed for a more detailed specification of LAI [31], but for flat deciduous leaves, this simplification is considered acceptable. Methodologies for the application of radiative transfer theory and modeling assumptions at laboratory and field scales in order to define the link between leaf reflectance and transmittance and canopy airborne hyperspectral data acquired with the compact airborne spectrographic imager (CASI) are discussed in the following sections. Airborne data acquired with different spectral and spatial characteristics over twelve Acer saccharum M (sugar maple) study sites in four consecutive years at $1 \mathrm{~m}, 2 \mathrm{~m}$, and $3 \mathrm{~m}$ spatial resolutions and in 72 spectral channels in the visible and NIR facilitated the investigation of such important questions through leaf and canopy radiative transfer models for chl $_{a+b}$ estimation.

\section{Methodologies for Estimation of Pigment Content in Vegetation Canopies: Scaling Up AND MOdel INVERSION}

Predictions of chlorophyll content or any other canopy biophysical parameter from airborne or satellite canopy reflectance can been carried out through four different methodologies: 1) directly studying the statistical relationships between ground-measured biochemical data and canopy-measured reflectance [19], [20]; 2) applying the leaf-level relationships derived between optical indices and the pigment content directly to canopy-measured reflectance [21]-[23]; 3) scaling up the leaf-level relationships based on optical indices related to pigment content through models of canopy reflectance or infinite reflectance $\left(R_{\infty}\right)$ [8], [15]-[17]; and 4) inverting the observed canopy reflectance through a canopy reflectance or infinite reflectance model coupled with a leaf model to estimate the optimum pigment content [17], [24]-[29].

The four proposed methodologies have advantages and disadvantages that are related to the complexity of the modeling approach selected and the degree of general or local applicability of the methodology in remote sensing. The first method studies the correlations between canopy-measured reflectance by a field, airborne or satellite sensor with ground-measured pigment, or any other biophysical constituent. In this case, no leaf reflectance is measured, and therefore, the link between canopy reflectance and biochemical content is found through statistical relationships. Multivariate analysis between visible infrared imaging spectrometer (AVIRIS) reflectance and total nitrogen, lignin, starch, chlorophyll content, and LAI [19] and with nitrogen and chlorophyll [20] applied by stepwise multiple-regression procedure using the AVIRIS spectral bands showed good statistical relationships derived at specific wavebands. Although significant correlations were found, no predictive capabilities could be inferred to other study sites since the locally-derived relationships are affected by species and canopy structure.

The second method, which uses statistical leaf-level relationships applied to canopy reflectance for pigment estimation, is also site and species specific [32], [33] and therefore requires relationship calibration that is a function of the canopy structure and viewing geometry at the time of remote sensing data collection. Therefore, the statistical relationships derived at leaf level need to be "calibrated" in order to be useful for estimation at the canopy level, due to the differences between the two media: one where the relationship is derived (leaf) and the other where it is applied for estimations (forest canopy). This methodology allows the derivation of relationships based on optical indices calculated at wavelengths where subtle changes in leaf reflectance correspond to specific biophysical processes that are targeted for measurement at the canopy level. Stepwise multiple-regression is often used to develop predictive algorithms from leaf reflectance, which are then applied to airborne data: AVIRIS [23] and airborne imaging spectrometer (AIS) spectra [21]. Laboratory canopy studies [22] and those using AVIRIS spectra [34] were directed to the identification of spectral bands at both leaf and canopy levels which are less sensitive to changes between levels, thereby minimizing effects due to the canopy, 
thus selecting spectral bands that could be used directly for prediction at canopy level. Application of leaf-level relationships to canopy reflectance through optical indices has been the traditional method used in the past, and a summary of the optical indices derived at the leaf level was described in the introduction.

In the third methodology, the same relationships between leaf constituent content and canopy reflectance are derived by scaling up the optical indices through infinite or canopy reflectance models [8], [9], [17]. A primary advantage is that the use of infinite or canopy reflectance models as part of the calculation of relationships avoids the post-calibration step to compensate for canopy structure or viewing geometry. Therefore, scaled-up leaf-level relationships can be used directly for bioindicator predictions on measured canopy reflectance data by considering canopy structure and viewing geometry information in the model scaling-up step. The objective of this method is the derivation of predictive algorithms to be used under certain canopy assumptions, not simply the evaluation of statistical correlations between sensor reflectance and ground measurements. In closed dense vegetation canopies, the reflectance canopy model can sometimes be replaced by different infinite reflectance formulations, as explained later, therefore simplifying the need for input parameters defining structure and geometry. As in the first methodology, this approach enables a search for subtle changes in leaf reflectance due to specific biophysical processes, and the reflectance model permits direct prediction of the canopy biochemical parameter. The main disadvantage is the requirement for leaf sample collection for the derivation of leaf-level relationships.

The fourth methodology, inversion of a canopy reflectance model coupled with a leaf model, attempts to avoid the development of leaf-level relationships through the use of a leaf model. In this approach [24]-[27], [29], the leaf radiative transfer simulation uses leaf biochemical constituents as input to model leaf reflectance and transmittance that is in turn used as input for the canopy reflectance model. The main advantage of this approach is that no leaf sample collection is needed to derive relationships, but suffers from the constraint that only biophysical parameters considered in the leaf model can be estimated from measured canopy reflectance. No subtle changes due to specific functioning effects can be sought, and therefore no changes at specific absorption wavelengths due to chlorophyll degradation at different senescence stages can be studied. That is, it is implicitly assumed that the leaf model captures all actual radiative processes accurately. Furthermore, the method is computational intensive, and no validation has been found in the literature reporting results in forest canopies from airborne or satellite-measured reflectance with ground truth; previous work has focused on synthetic data [24], [28], field spectrometer data [25], estimation of results with no validation due to a lack of ground truth [26], comparison of different model inversion techniques using simulated data [35], and simulation studies modeling three-dimensional (3-D) canopies used for applying inversion techniques [27], [28]. Successful $\mathrm{chl}_{a+b}$ and LAI estimation results were reported for agricultural crops observed with airborne CASI data in which comparisons of inversions from four radiative transfer models coupled to PROSPECT [29], using Minolta SPAD-502 for estimation of leaf $\mathrm{chl}_{a+b}$, are described. Other studies with lower spatial and spectral resolution data [36], [37] use canopy model inversion for extracting canopy biophysical information from large swath satellite data at global scales using the advanced very high resolution radiometer (AVHRR) and VEGETATION/SPOT4, respectively, and therefore, its applicability and portability to narrow-band hyperspectral high-spatial airborne data cannot be evaluated.

Simulation of the tree crown reflectance spectral content for comparison to the measured canopy reflectance and retrieved optical indices may be done through $R_{\infty}$ and CR models, depending upon the complexity and assumptions made with respect to the type of vegetation canopy and viewing geometry. Infinite reflectance formulations model reflectance without canopy structure or viewing geometry considerations, based solely on leaf reflectance and transmittance. These formulations correspond to optically thick leaf material with different assumptions for the multiple scattering between leaf layers. This thick-leaf or leaf-stack concept may have applicability to closed deciduous canopies characterized generally by high LAI, therefore with little effect of soil background and understorey. CR models, such as SAILH [38] and MCRM [39], [40] used in this research, on the other hand, take into account viewing geometry and canopy structure, therefore modeling those effects in the canopy reflectance by different approximations generally based on the RTE and geometrical optical considerations.

Different infinite reflectance formulations have been derived based on assumptions related to the scattering between layered leaves forming the optically thick canopy. In each case, the reflectance for an optically thick medium is expressed in terms of the inherent single leaf reflectance and transmittance. Lillestaeter [41] derived a simple formulation (referred to here as $R_{\infty 1}$ ) from measurements of leaf-stack apparent reflectance over known dark and bright backgrounds, ignoring multiple scattering, and considering equal reflectance for both sides of the leaf (1a). This simple formulation was found inadequate by Miller et al. [42] to simulate the measured reflectance of leaf stacks. A matrix formulation by Yamada and Fujimura [43] was used in a simulation that included multiple reflectance between leaves and considering different adaxial and abaxial reflectance for the leaf $\left(R_{\infty 2}\right.$, (1b). The Hapke [44] infinite reflectance formulae $\left(R_{\infty 3}\right)$ corresponds to a medium with a single scattering albedo $\omega_{\mathrm{o}}$ assumed approximately equal to reflectance $(\rho)+$ transmittance $(\tau)$ for a pile of leaves, $(1 \mathrm{c})$. The corresponding formulae approximating thick leaf canopies are

$$
\begin{aligned}
R_{\infty 1} \text { approx. leaf stack } \rightarrow R_{\infty} & =\frac{\rho}{1-\tau^{2}} \\
R_{\infty 2} \text { leaf stack } \rightarrow R_{\infty} & =\frac{\rho}{1-\frac{2 \tau^{2}}{1+\left(1-4 \tau^{2}\right)^{\frac{1}{2}}}} \\
R_{\infty 3} \text { thick leaf } \rightarrow R_{\infty} & =\frac{1-\alpha^{\frac{1}{2}}}{1+\alpha^{\frac{1}{2}}}
\end{aligned}
$$

Both CR and $R_{\infty}$ models have been used in this research for $\mathrm{chl}_{a+b}$ estimation from airborne hyperspectral data collected over closed Acer saccharum M. forest canopies using the 


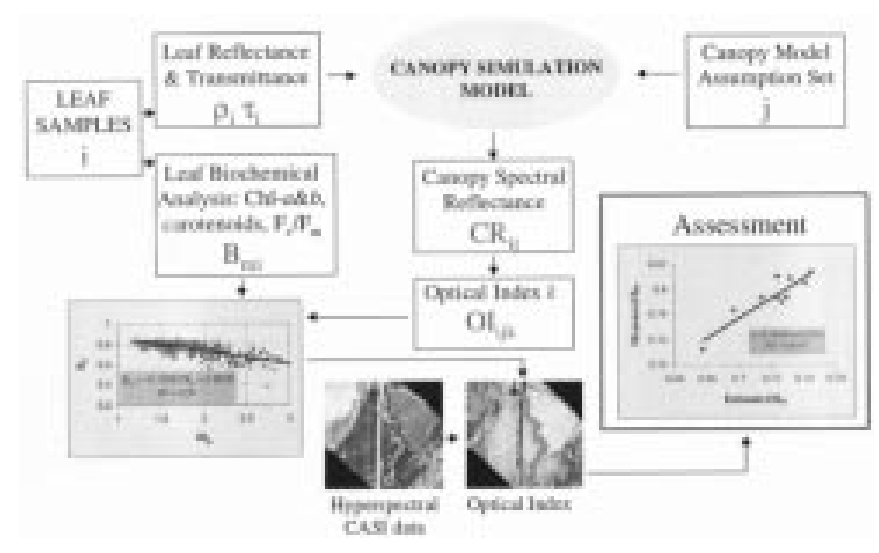

Fig. 1. Schematic view of the overall analysis methodology followed for the scaling-up method. Leaf-level reflectance and transmittance measurements are scaled-up to canopy level through infinite and CR models and input parameters related to the canopy structure and viewing geometry. Relationships between optical indices calculated from above-canopy simulated reflectance and ground-truth bioindicators are applied to above-canopy hyperspectral CASI reflectance to obtain bioindicator estimation. Assessment is made comparing ground-truth measured with estimated bioindicators.

scaling-up and numerical model inversion methods explained below.

\section{A. Parameter Estimation by Scaling-Up Optical Indices}

A schematic view of the approach used here for estimation of leaf bioindicators through the scaling-up of leaf-level optical indices is shown in Fig. 1. Single leaf reflectance and transmittance measurements $\left(\rho_{i}, \tau_{i}\right)$ from field data sampling are used for the simulation of above-canopy reflectance through infinite reflectance and canopy reflectance models, constrained by a specific canopy model parameter assumption set $(j)$. A specific set of assumed input parameters to the CR model defines the canopy structure, by a more or less complex set of canopy parameters, and the viewing geometry, defined by the solar zenith and azimuth, and viewing angles, needed for simulating canopy reflectance from single leaf reflectance and transmittance measurements. Canopy spectral reflectances (denoted $C R_{\mathrm{ij}}$ ), or more precisely the above-canopy spectral bidirectional reflectance factor, simulated through the canopy simulation model are used to calculate specific optical indices $k$ (denoted $O I_{\mathrm{k}}$ ). For a given optical index, a set of $n$ values are calculated from the leaf-level spectral measurements used for CR simulation. Leaf bioindicators (denoted $B_{\mathrm{mi}}$ ) measured in each leaf sample ( $\mathrm{chl}_{a+b}$, carotenoids, etc) are used to derive relationships with the optical indices $O I_{\mathrm{ijk}}$ calculated from the above-canopy simulated spectra. Therefore, the relationship between a given bioindicator (e.g., $\mu \mathrm{g} \mathrm{chl}_{a+b} / \mathrm{cm}^{2}$ ) and a given optical index (e.g., $R_{750} / R_{710}$ ) is calculated from simulated canopy reflectance rather than from leaf-level measured reflectance. This relationship is therefore affected by canopy structural parameters and viewing geometry, which permits its application to above-canopy measured reflectance. Thus, the relationships between the set of optical indices $O I_{\mathrm{ijk}}$ and the set of bioindicators $B_{\mathrm{mi}}$ are then applied to hyperspectral CASI reflectance data to obtain bioindicator estimations. To do so, above-canopy measured CASI reflectance is used to calculate the CASI-optical indices input using relationships of the form
$B_{\mathrm{mi}}=f\left(O I_{\mathrm{ijk}}\right)$, e.g., $\mu \mathrm{g} \mathrm{chl}_{a+b} / \mathrm{cm}^{2}=a \cdot R_{750} / R_{710}+b$, with $a$ and $b$ constant parameters for above-canopy simulation, and $R_{750} / R_{710}$ an optical index derived from the above-canopy reflectance. This methodology enables the direct application of sensor-derived optical indices in scaled-up algorithms that are therefore a function of the canopy structure and viewing geometry, precluding the need for calibration of prediction relationships. Assessment of optical indices as estimators of bioindicators is then made comparing in-field measured bioindicators (measured $B_{m}$ ) with CASI-derived estimations (estimated $B_{m}$ ).

\section{B. Parameter Estimation by Model Inversion}

The estimation of a biophysical canopy parameter by numerical model inversion can generally be carried out by different methodologies: 1) look-up tables (LUT); 2) iterative optimization (OPT); and 3) neural networks (NNT). The look-up table technique is conceptually the simplest [35] and consists of the generation of an output table for a discrete set of input parameters covering the expected range of the parameters. The table is used to find the measured value that is directly related to a given set of input parameters. This method requires the generation of large number of cases that are subsequently used to compare with measured data. Iterative Optimization is the classical technique for inverting radiative transfer models in remote sensing [25], [26], [45], [46] and consists of minimizing a function that calculates the root mean square error (RMSE) between the measured and estimated quantities by successive input parameter iteration. Neural networks are nonphysical methods that relate a set of input variables to a set of output variables by a learning process and have been shown to be efficient in inversion of canopy models [47], [48].

Iterative-optimization numerical model-inversion techniques to estimate chlorophyll content using a coupled leaf model and a canopy model requires three consecutive steps: 1 ) estimation of leaf reflectance and transmittance $(\rho, \tau)$ from a set of leaf model input parameters such as the parameter to be estimated, $\mathrm{chl}_{a+b}$, and other leaf cellular structural or scattering parameters; 2) estimation of canopy reflectance from leaf-level, model-estimated $\rho, \tau$, and set of canopy model parameters that define canopy structure and viewing geometry; and 3) error calculation by comparison of estimated canopy reflectance $\rho^{*}$ to the at-sensor measured reflectance $\rho_{m}$. Error calculation consists in determining the set of parameters $P=\left(\mathrm{N}, \mathrm{Chl}_{a+b}, C_{\mathrm{w}}, L A I, \theta_{\mathrm{s}} \ldots\right.$, which minimizes a merit function $\Delta^{2}$ over the whole spectrum

$$
\Delta^{2}=\sum_{n}\left[\rho_{m}\left(\lambda_{i}\right)-\rho^{*}\left(\lambda_{i}, P\right)\right]^{2}
$$

where $\rho_{m}\left(\lambda_{i}\right)$ is the measured canopy spectral reflectance, and $\rho^{*}\left(\lambda_{i}, P\right)$ is the modeled canopy spectral reflectance with a set of $P$ parameters. Different merit functions have been defined in the literature, each based on different assumptions. The minimizing function for numerical model inversion using reflectance data in several spectral bands can be calculated 1) from single reflectance channels, comparing the estimated with the measured reflectance in all spectral bands [(2), [24], [25]; 2) using $w_{i}$ 
weighting factors that represent the weight given to the $i$ th wavelength. The usual protocol is to choose weighting coefficients $w_{i}$ to be proportional to the inverse of the measured canopy reflectance $w_{i}=1 / \rho_{m, i}, i$, thereby placing more weight to wavelengths in the visible part of the spectrum where pigment absorption is maximum, and minimizing the impact of errors between measured and estimated reflectance in the NIR, where chlorophyll absorption decreases and reflectance is driven by canopy structure; 3) by a more sophisticated construction of merit functions [26], where penalization to the merit function [49] is introduced if the best fit is found when a parameter being inverted falls outside the prior-established range of allowed values; and 4) building merit functions based on spectral transforms or vegetation indices [46], in which the merit function generated is based on the optical index that is supposed to be related with the parameter subject to estimation, in this case $\mathrm{chl}_{a+b}$.

As an example, (3) presents a merit function when the red-edge spectral parameter $R_{750} / R_{710}$ is used for pigment estimation, which could easily be modified if a combination of optical indices is used

$$
\Delta^{2}=\left[\left(\frac{R_{750}}{R_{710}}\right)_{m}-\left(\frac{R_{750}}{R_{710}}, P\right)_{*}\right]^{2}
$$

where $\left(R_{750} / R_{710}\right)_{m}$ is the optical index calculated from measured canopy reflectance, and $\left(R_{750} / R_{710}, P\right)_{*}$ is the optical index calculated from modeled canopy reflectance for a given set of input parameters $P$. The use of optical indices in the merit function has not been found reported in any of the validation work found in the literature, in spite of the significant inherent potential of this approach for remote sensing applications. Reflectance values measured from airborne or satellite sensors are a function of illumination, canopy structure, and atmospheric condition at the time of data collection. On the other hand, estimation of biophysical parameters through optical indices maximizes the sensitivity to such biophysical parameters, while normalizing external effects due to atmosphere, illumination conditions, and viewing geometry [50], [51]. Therefore in this research, leaf-level optical indices and ratios that showed good correlation with pigment content are proposed here to be used as a basis for the merit function for model inversion, as discussed later.

These different approaches have been tested in this research, in order to compare the pigment estimation by different techniques using hyperspectral airborne data collected in 1998, 1999, and 2000. This data set provides a valuable validation database for model inversion with hyperspectral data in closed dense maple canopies. The experimental methods and materials used to carry out the pigment investigations are described below.

\section{MethodS AND EXPERIMENTAL DATA}

CASI airborne hyperspectral data were collected in deployments over 12 sites of Acer saccharum M. in the Algoma Region, ON, Canada, in 1997, 1998, and 1999. A validation of the methodologies developed with 1997, 1998 and 1999 data over the 12 study sites was carried out in June 2000 selecting a different set of 14 plots of the same species. The above-canopy data acquisition using the CASI sensor was divided into three missions, each with a specific sensor mode of operation: the Mapping Mission, with $0.5 \mathrm{~m}$ spatial resolution and seven spectral bands (Fig. 2); the Hyperspectral Mission, with $2 \times 4 \mathrm{~m}$ spatial resolution, 72 channels and $7.5 \mathrm{~nm}$ spectral resolution (Fig. 3), and the Full-Spectral Hyperspectral Mission, with 288 channels and $2.5 \mathrm{~nm}$ spectral resolution. The 12-bit radiometric resolution data collected by CASI were processed to at-sensor radiance using calibration coefficients derived in the laboratory by the Centre for Research in Earth and Space Technology (CRESTech). Aerosol optical depth data at 340, 380, 440, 500, 670,870 , and $1020 \mathrm{~nm}$ were collected using a Micro-Tops III sunphotometer in the study area at the time of data acquisition in order to derive aerosol optical depth at $550 \mathrm{~nm}$ to be used to process image data to ground-reflectance using the CAM5S atmospheric correction model [52]. Reflectance data were georeferenced using GPS data collected onboard the aircraft. Final registration of the hyperspectral mode imagery was achieved by registration to the CASI mapping mission imagery using visual identification of ground-referenced $1 \mathrm{~m}$ white targets, which served to accurately identify the location of the sites.

Mean reflectance values per plot were calculated from the hyperspectral imagery in each Acer saccharum M. study site of $20 \times 20 \mathrm{~m}$. The mean reflectance per plot was calculated selecting the $25 \%$ of pixels with highest reflectances in the NIR, therefore targeting crowns while minimizing the influence of shadows, canopy openings and the direct understorey reflectance. The study sites of sugar maple were selected in 1997 from existing provincial plot networks in the Algoma Region, representing a range of productivity and decline. In particular, six permanent sample plots from the provincial Growth and Yield Program [53], [54] were chosen to investigate the effects of stand productivity in maple. Another six plots were selected from the provincial Hardwood Forest Health Network [55], [56] to represent a gradient in maple forest decline. Detailed stand records exist and these sites are considered representative of tolerant hardwood forests in the Algoma Region.

A field sampling campaign was carried out for biochemical analysis of leaf chlorophyll concentration, along with leaf reflectance and transmittance within the same period of the field data acquisition. Samplings were carried out in June and July of 1998 and 1999, and in June 2000, collecting from the top of the crowns at each one of the twelve Sugar Maple study sites. Four leaves per tree with five trees per study site were sampled for measurements of $\mathrm{chl}_{a+b}$ and spectral measurements of reflectance and transmittance, collecting a total of 440 leaf samples per year. Pigment content measurements from the leaves were made as in [15], [16]. Biochemical analysis of samples from 2000 showed a narrower range of $\mathrm{chl}_{a+b}$ content compared to the 1998 and 1999 sites, with values falling into the $29.8-42.7 \mu \mathrm{g} / \mathrm{cm}^{2}$ interval (while in previous years, ranges were $19.1-41.1 \mu \mathrm{g} / \mathrm{cm}^{2}$ in 1998 , and $26.6-45.8 \mu \mathrm{g} / \mathrm{cm}^{2}$ in 1999). LAI measurements were acquired for all the plots using a PCA Li-Cor 2000 instrument.

Single leaf reflectance and transmittance measurements were acquired on all leaf samples using a Li-Cor 1800-12 integrating 


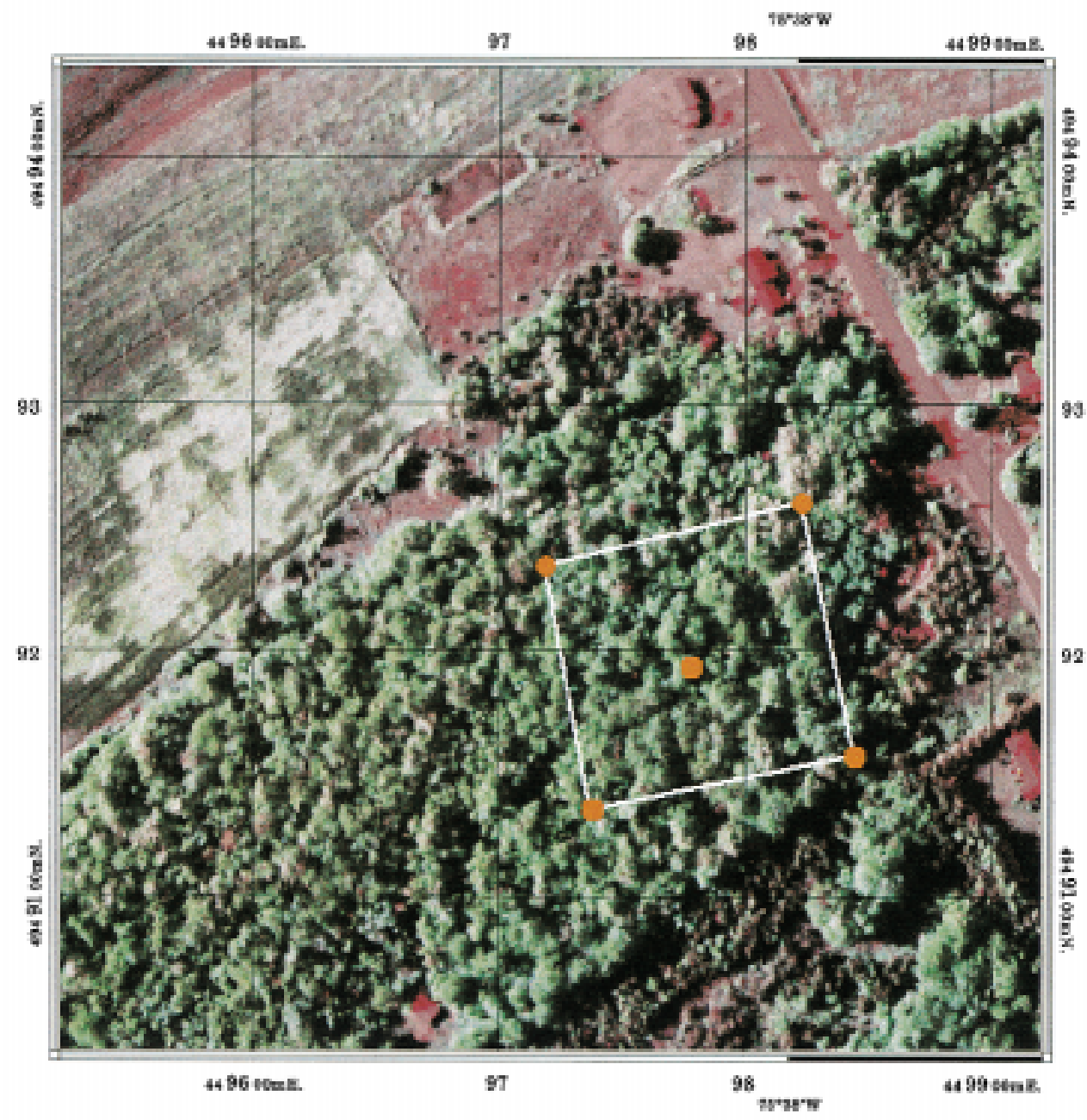

oTM as wos aakd taps!

Fig. 2. CASI image collected over one of the Acer saccharum M. study sites in the mapping mission mode of operation, with seven channels and $0.8 \mathrm{~m}$ spatial resolution. The high spatial resolution facilitated target location needed in the image registration process, therefore, locating the study site of $20 \times 20 \mathrm{~m}(1998$ and $1999)$ and $80 \times 80 \mathrm{~m}$ blocks (2000 validation campaign, white box in figure).

sphere apparatus coupled by a $200 \mu \mathrm{m}$ diameter single mode fiber to an Ocean Optics model ST 1000 spectrometer with a 1024 element detector array, yielding a $0.5 \mathrm{~nm}$ sampling interval and $\sim 7.3 \mathrm{~nm}$ spectral resolution in the $340-860 \mathrm{~nm}$ range. The spectrometer is controlled and read out by a National Instruments multipurpose data acquisition card (DAC-550). Software was designed to allow detailed control of signal verification, adjustment of integration time, and data acquisition [57]. Spectral bandpass characterization performed using a mercury spectral line lamp source yielded full-width at half maximum (FWHM) bandwidth estimates of $7.37 \mathrm{~nm}, 7.15 \mathrm{~nm}$, and 7.25 $\mathrm{nm}$, at $438.5 \mathrm{~nm}, 546.1 \mathrm{~nm}$ and $576.9 \mathrm{~nm}$, respectively. Fiber spectrometer wavelength calibration was performed using the Ocean Optics HG-1 mercury-argon calibration source, which produces $\mathrm{Hg}$ and $\mathrm{Ar}$ emission lines between 253 and $922 \mathrm{~nm}$. Single leaf reflectance and transmittance measurements were acquired following the methodology described in the manual for the Li-Cor 1800-12 system [58] in which six signal measurements are required (see [15], [16] for measurement protocol). An integration time of $609.3 \mathrm{msec}$ was used for all sample mea- surements. Smoothing of reflectance and transmittance was carried out as described in [15], using a Savitzky-Golay approach with a third-order polynomial function with $25 \mathrm{~nm}$ bandwidth found optimum for our spectral data set.

\section{RESULTS}

Leaf-level relationships were developed between narrow-band optical indices and $\mathrm{chl}_{a+b}$ from the samples collected from the Acer saccharum M. study sites in June and July 1998 and 1999 campaigns. Red edge and spectral and derivative indices consistently showed the best relationships in the two-year study, demonstrating that $\left(R_{750} / R_{710}\right)(r=0.92), \operatorname{Vog} 1\left(R_{740} / R_{720}\right)(r=0.91)$, $\operatorname{Vog} 2\left(R_{734}-R_{747}\right) /\left(R_{715}+R_{726}\right)(r=0.90), \operatorname{Vog} 3$ $\left(R_{734}-R_{747}\right) /\left(R_{715}+R_{720}\right)(r=-0.91), \operatorname{Vog} 4$ $\left(D_{715} / D_{705}\right)(r=0.90)$, GM1 $\left(R_{750} / R_{550}\right)(r=0.83), \mathrm{GM} 2$ $\left(R_{750} / R_{700}\right)(r=0.91)$ and $\mathrm{Ctr} 2\left(R_{695} / R_{760}\right)(r=-0.81)$ (red-edge indices) and $\lambda_{\mathrm{p}}(r=0.90)$, DP21 $\left(D \lambda_{\mathrm{p}} / D_{703}\right)(r=$ $0.78)$, and DP22 $\left(D \lambda_{\mathrm{p}} / D_{720}\right)(r=-0.76)$ (spectral and 

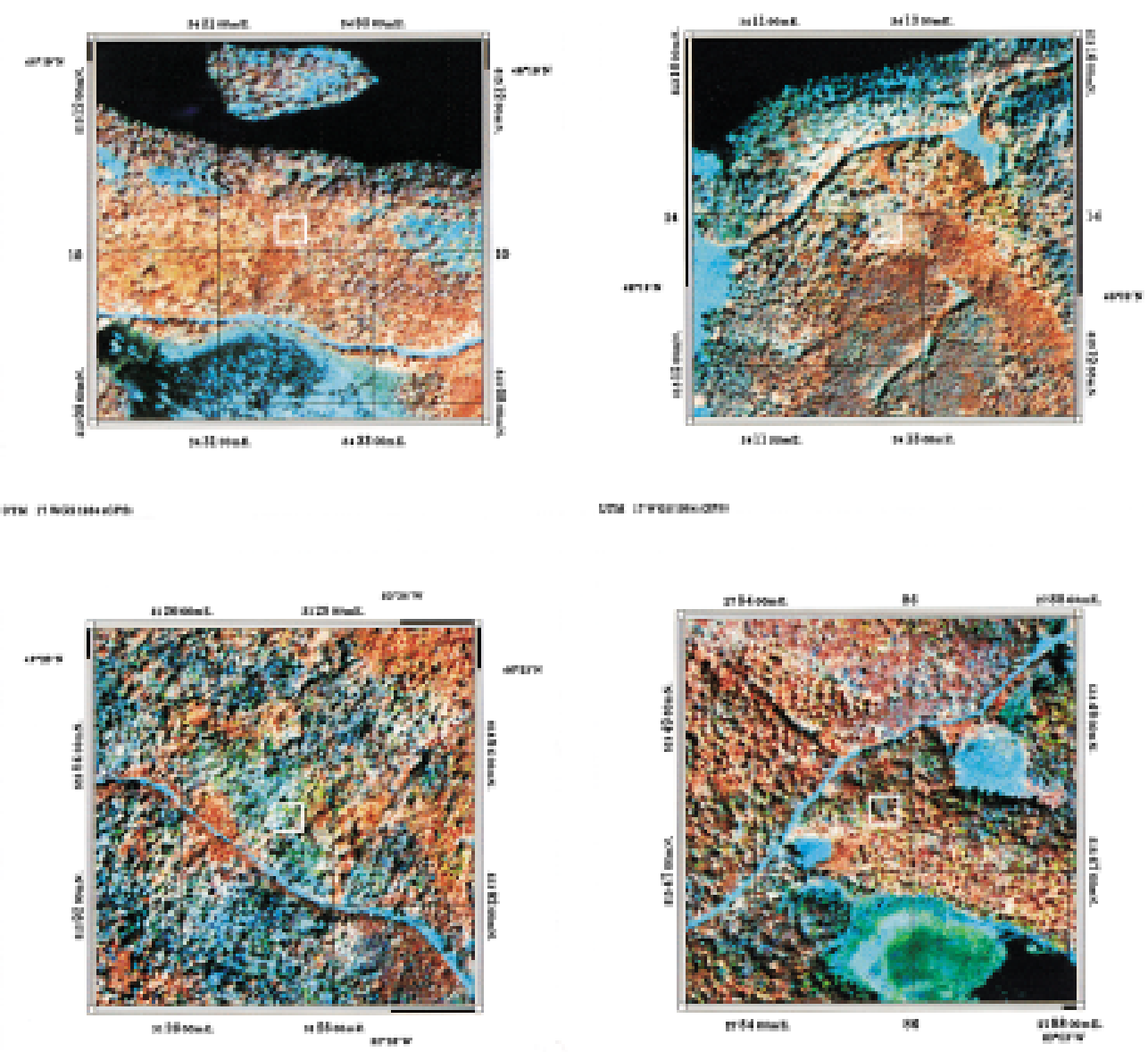

me nom raumen
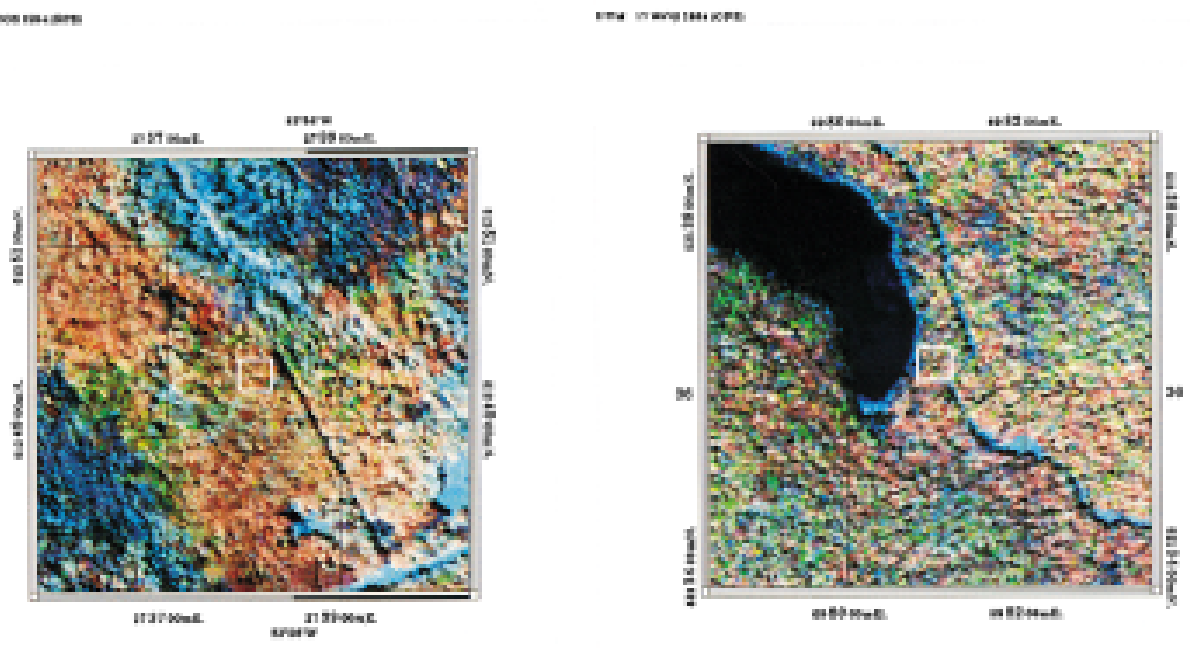

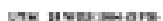

Fig. 3. View of a selection of the study areas of $500 \times 500 \mathrm{~m}$ acquired over the maple decline plots used for field measurements in 1997, 1998, and 1999. CASI data of $2 \times 4 \mathrm{~m}$ and 72 channels from the study sites for leaf sampling collection (in white box). Composite image from $555.6 \mathrm{~nm}$ (blue), $706.8 \mathrm{~nm}$ (green), and $852.1 \mathrm{~nm}$ (red).

derivative indices) achieved the best results in both early and mid summer when used for $\mathrm{chl}_{a+b}$ estimation. Optical indices calculated from the red edge are consistently well correlated with $\operatorname{chl}_{a+b}$, since this is the spectral region where pigment absorption decreases, therefore exhibiting increasing effects of the medium structure in the measured reflectance, affecting the slope. Results obtained with airborne CASI reflectance data from the study sites for the estimation of $\mathrm{chl}_{a+b}$ by the scaling-up through a radiative transfer model and by model inversion of a coupled leaf and canopy model approaches are shown in next sections.

\section{A. Estimation of $\mathrm{chl}+b$ by Scaled-up Optical Indices Applied To CASI Hyperspectral Data}

The leaf-level relationships between optical indices and $\mathrm{chl}_{a+b}$ calculated from single leaf reflectance and trans- 
TABLE I

DETERMINATION COEFFICIENTS AND RMSE $\left(\mu \mathrm{g} / \mathrm{cm}^{2}\right)$ OBTAINED IN $\mathrm{ch}_{a+b}$ ESTIMATIONS APPLYING RELATIONSHIPS FROM SiNGLE LEAF REFLECTANCE SET OF

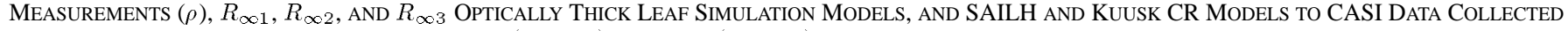
OVER ACER SACCHARUM M. STUDY SITES IN $1998(n=12)$ AND $1999(n=12)$. CODES: VisIBLE RATIOS (C1), RED-EDGE INDICES (C2), AND SPECTRAL AND DERIVATIVE INDICES (C3). IN GREY RELATIONSHIPS WITH $R^{2}>0.3$ AND RMSE $<10$

\begin{tabular}{|c|c|c|c|c|c|c|c|c|c|c|c|c|c|c|}
\hline & \multicolumn{2}{|c|}{$\begin{array}{c}\text { Determination } \\
\text { Coefficient }\end{array}$} & \multicolumn{6}{|c|}{$\begin{array}{c}\text { RMSE }\left(\mu \mathrm{g} / \mathrm{cm}^{2}\right) \\
1998\end{array}$} & \multicolumn{6}{|c|}{$\begin{array}{c}\text { RMSE }\left(\mu \mathrm{g} / \mathrm{cm}^{2}\right) \\
1999\end{array}$} \\
\hline & $\begin{array}{c}R^{2} \\
1998\end{array}$ & $\begin{array}{c}\mathrm{R}^{2} \\
1999\end{array}$ & $\rho$ & $\mathbf{R}_{\infty 1}$ & $\mathbf{R}_{\infty 2}$ & $\mathbf{R}_{\infty 3}$ & $\mathbf{R}_{\mathrm{SAIL}}$ & $\mathbf{R}_{\text {Kuusk }}$ & $\rho$ & $\mathbf{R}_{\infty 1}$ & $\mathbf{R}_{\infty 2}$ & $\mathbf{R}_{\infty 3}$ & $\mathbf{R}_{\text {SAII. }}$ & $\mathbf{R}_{\text {Kutusk }}$ \\
\hline$\overline{\mathbf{R}_{750} / \mathbf{R}_{710(\mathfrak{c} 2)}}$ & 0.48 & 056 & 21.67 & 905 & 588 & 811 & 10.78 & 12.09 & 24.68 & 853 & 425 & 8.27 & 11.39 & 10.41 \\
\hline $\operatorname{Vog} 1_{(\mathrm{c} 2)}$ & 0.56 & 0.46 & 27.36 & 14.2 & 794 & 576 & 8.03 & 9.75 & 37.49 & 17.24 & 4.35 & 5.32 & 7.66 & 926 \\
\hline $\mathbf{G}_{-} \mathbf{M} 2_{(\mathrm{c} 2)}$ & 0.37 & 0.56 & 22.84 & 10.86 & 644 & 8.03 & 11.91 & 12.32 & 18.08 & 485 & 5.62 & 9.91 & 13.82 & 11.46 \\
\hline $\operatorname{Vog} 3_{(c 2)}$ & 0.55 & 0.56 & 34.62 & 15.5 & 5.29 & 795 & 8.83 & 10.48 & 41.72 & 14.72 & 4.75 & 7.84 & 9.99 & 7.56 \\
\hline$\lambda_{p(\mathrm{c} 3)}$ & 0.53 & 0.52 & 10.18 & 5.75 & 7.46 & 21.12 & 25.73 & 31.71 & 11.17 & 4.78 & 64 & 14.23 & 49 & 5.43 \\
\hline $\operatorname{Vog} 2$ & 0.56 & 0.56 & 34.82 & 16.07 & 537 & 7.83 & 857 & 10.29 & 39.98 & 14.2 & 493 & 8.02 & 10.33 & 752 \\
\hline $\operatorname{Vog} 4_{(\mathrm{c} 2)}$ & 036 & 0.66 & 857 & 5.36 & 574 & 9.81 & 13.47 & 15.78 & 14.92 & 6.79 & 385 & 3.39 & 796 & 10.19 \\
\hline G_M1 $_{(\mathrm{c} 2)}$ & 0.29 & 0.5 & - & - & . & - & - & - & 54.56 & 28.81 & 13.14 & 416 & 6.46 & 6.12 \\
\hline Ctr2 (c2) & 0.38 & 0.52 & 17.97 & 11.51 & 7.52 & 8.9 & 15.01 & 14.38 & 16.3 & 6.65 & 565 & 13.11 & 22.74 & 16.55 \\
\hline DP21 (c3) & 0.58 & 0.48 & 32.19 & 12.74 & 7.48 & 4.92 & 7.02 & 8.55 & 46.92 & 18.84 & 4.76 & 3.95 & 5.61 & 6.25 \\
\hline Ctr1 (c1) & 0.16 & 0.29 & . & - & & - & 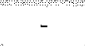 & - & - & - & 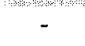 & - & - & 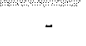 \\
\hline DP22 (c3) & 0.42 & 0.16 & 8.23 & 13.45 & 9.98 & 8.13 & 11.24 & 659 & - & - & - & - & - & - \\
\hline Lic4 (c1) & 0.07 & 0.49 & - & - & - & - & - & - & 27.24 & 27.07 & 27.06 & 683 & 10.61 & 11.03 \\
\hline $\mathbf{G}_{(\mathrm{cl})}$ & 0.26 & 0.38 & - & - & - & - & - & - & 5.58 & 499 & 407 & 15.67 & 7481 & 486 \\
\hline
\end{tabular}

mittance data collected from the ground-truth deployments in June and July 1998 and 1999, and those scaled-up to above-canopy level through infinite reflectance models, and SAILH and Kuusk canopy reflectance models were applied to CASI hyperspectral data for $\mathrm{chl}_{a+b}$ estimation. For the application of the SAILH and Kuusk canopy reflectance models nominal input parameters derived from the study areas were: $\mathrm{LAI}=3.5$, plagiophile leaf angle distribution function (LADF), soil reflectance data derived from CASI imagery, and model-estimated skylight irradiance fraction based on conditions during airborne acquisitions. Additional parameters needed in the Kuusk model were $n=1.4, s / l=0.007$ and $\theta^{*}=40^{\circ}$, and $\epsilon=0.95$, and $\theta_{m}=45^{\circ}$ for the LADF assumed to be a plagiophile distribution. A model parameter sensitivity study was carried out in order to study the effect of such nominal structural and viewing geometry parameters on the optical indices that are used for prediction. This sensitivity study [9], [17] showed that low LAI values are very critical to the accuracy of predicted bioindicator through optical indices regardless of the considered type of canopy. The study showed that differences between the predicted bioindicator using nominal canopy parameters and the prediction with variable $\theta_{\mathrm{s}}$ and LADF is insignificant (less than 5\%) when LAI is higher than three, and the optical indices used are the red edge reflectance-ratio indices and spectral and derivative red edge indices. Furthermore, the canopy type was shown to be irrelevant when the same optical indices are used (less than $5 \%$ difference) for the estimations when LAI is higher than three and the $\theta_{\mathrm{s}}$ is a nominal $30^{\circ}$. It was shown that derivative indices are less sensitive to low LAI values than other optical indices, demonstrating that red edge and derivative indices are more suitable for bioindicator prediction and mapping with high spatial hyperspectral remote sensing data. Results also demonstrated the small effect of the solar zenith angle $\theta_{\mathrm{s}}$, especially in red edge spectral and derivative indices, with less than $2 \%$ variation in the predicted bioindicator when $\theta_{\mathrm{s}}$ changes from $20^{\circ}$ to $60^{\circ}$, where the optical indices used are Vogelmann $\left(R_{740} / R_{720}\right)$ and DP21 $\left(D \lambda_{\mathrm{o}} / D_{703}\right)$, respectively. Therefore, changes in $\theta_{\mathrm{s}}$ from $29^{\circ}$ to $41^{\circ}$ in the $12 \mathrm{CASI}$ images obtained from the study sites in 1998 and 1999 are not expected to affect the bioindicator prediction when $\theta_{\mathrm{s}}=30^{\circ}$ was chosen as nominal input parameter in the CR model when scaling from leaf-level to canopy-level. Presented in Table I are the determination coefficients and RMSEs obtained in $\mathrm{chl}_{a+b}$ estimation by applying relationships obtained from single leaf reflectance set of measurements $(\rho)$, optically thick leaf simulation models ( $R_{\infty 1}, R_{\infty 2}$, and $R_{\infty 3}$ ), and CR models (SAILH and Kuusk) to CASI data collected over the Acer saccharum M. study sites for $1998(n=12)$ and $1999(n=12)$. These results show consistency between CASI-level and leaf-level relationships obtained between optical indices and $\mathrm{chl}_{a+b}$ content. The best indices for $\mathrm{chl}_{a+b}$ estimation that were found at leaf level are likewise those achieving best estimations when applied to CASI canopy reflectance. It is also demonstrated that a cross-seasonal consistency exists in the performance of the best indices for 1998 and 1999, showing that the good estimation performed by the best indices is maintained from 1998 to 1999 campaigns.

Red-edge indices, especially $\lambda_{\mathrm{p}}$ and DP21 $\left(D_{\lambda \mathrm{p}} / D_{703}\right)$ and spectral and derivative indices such as $\left(R_{750} / R_{710}\right)$, $\operatorname{Vog} 1 \quad\left(R_{740} / R_{720}\right)$, G_M2 $\left(R_{750} / R_{700}\right), \operatorname{Vog} 3\left(R_{734}-\right.$ $\left.R_{747}\right) /\left(R_{715}+R_{720}\right), \operatorname{Vog} 2\left(R_{734}-R_{747}\right) /\left(R_{715}+R_{726}\right)$, $\operatorname{Vog} 4 \quad\left(D_{715} / D_{705}\right), \quad$ G_M1 $\left(R_{750} / R_{550}\right)$, and Ctr2 $\left(R_{695} / R_{760}\right)$ are the best optical indices for $\mathrm{chl}_{a+b}$ estimation at canopy level. Other optical indices show significance when used as estimators of $\mathrm{chl}_{a+b}$, but inconsistency exists 

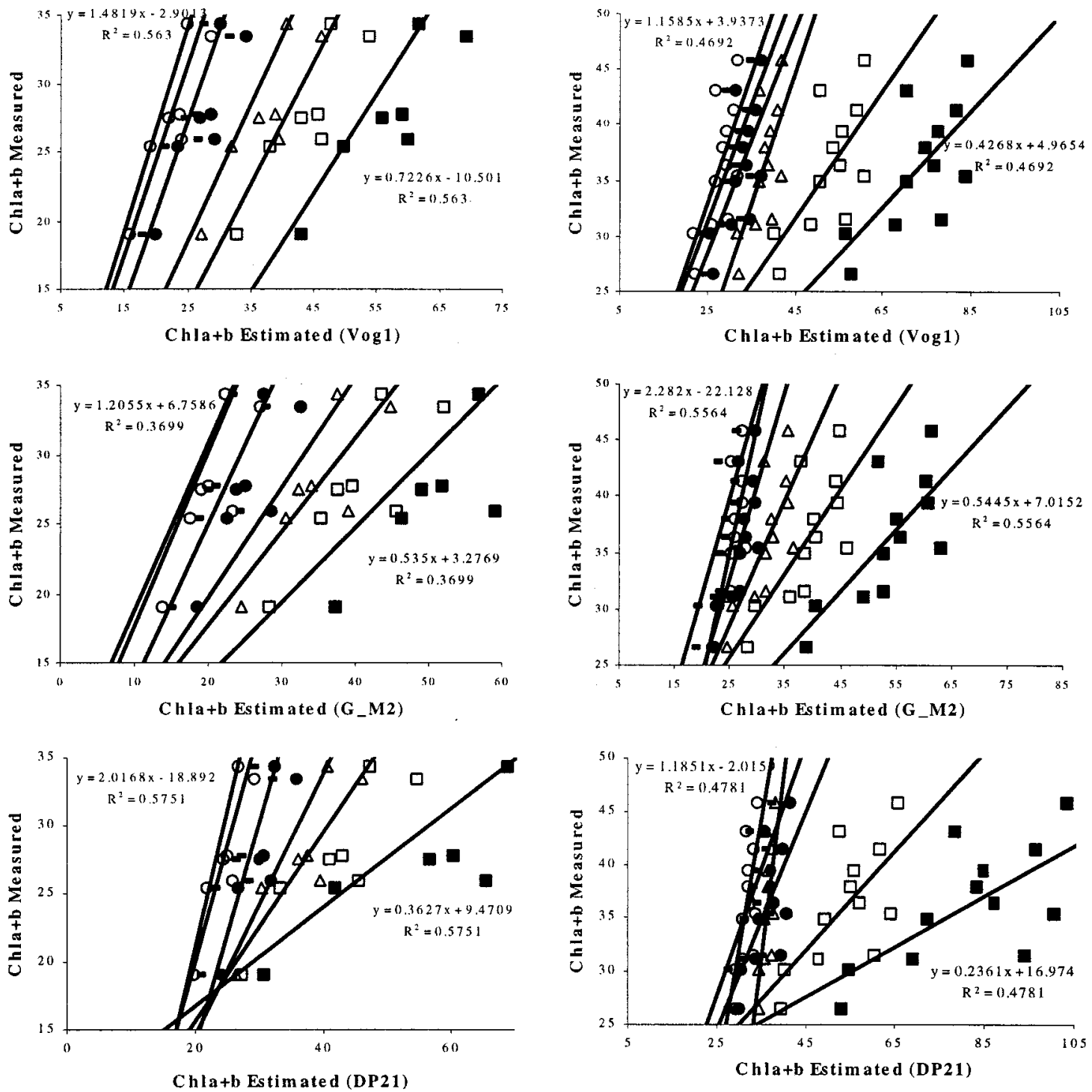

0 Kuusk - Sail $\square \operatorname{Rinf} 1 \Delta \operatorname{Rinf2} \bullet \operatorname{Rinf3} \square \operatorname{Rf} 1$

OKuusk - Sail $\square \operatorname{Rinf1} \Delta \operatorname{Rinf2} \bullet \operatorname{Rinf3} \square \operatorname{Rf1}$

Fig. 4. Estimation of $\mathrm{ch}_{a+b}$ from CASI data using Vog1 $\left(R_{740} / R_{720}\right)$, G_M2 $\left(R_{750} / R_{700}\right)$, and DP21 $\left(D_{\lambda \mathrm{p}} / D_{703}\right)$ optical indices developed at leaf level through $\rho, R_{\infty}$ and CR models in 12 Acer saccharum M. study sites for 1998 (left) and 1999 (right).

between deployments such as Lic4 (area under 450-680 region) $\left(R^{2}=0.06,1998 ; R^{2}=0.49,1999\right)$, PRI2 $\left(R_{550}-R_{531}\right) /\left(R_{550}+R_{531}\right)\left(R^{2}=0.32,1998 ; R^{2}=0.14\right.$, $1999)$, and $R_{\mathrm{o}}\left(R^{2}=0,1998 ; R^{2}=0.34,1999\right)$. Traditional and well accepted optical indices for indicators of vegetation status such as NDVI and SR performed poorly in the two consecutive years: $R^{2}=0,1998 ; R^{2}=0.05,1999$ (NDVI), and $R^{2}=0,1998 ; R^{2}=0.05,1999$ (SR). These traditional indices, calculated as ratios of NIR/VIS, are primarily tracking canopy structural changes but are not able to track subtle changes due to pigment content variation between study sites. Therefore, this study provides strong evidence that canopies with homogeneous structure but different chlorophyll content need the use of red edge and spectral indices to estimate changes in pigment content.

The estimation of $\operatorname{chl}_{a+b}$ from $\operatorname{Vog} 1\left(R_{740} / R_{720}\right), \mathrm{G}_{-} \mathrm{M} 2$ $\left(R_{750} / R_{700}\right)$ and DP21 $\left(D_{\lambda \mathrm{p}} / D_{703}\right)$ optical indices using $\rho$,
$R_{\infty 1}, R_{\infty 2}$, and $R_{\infty 3}$, and the SAILH and Kuusk CR models are illustrated in Fig. 4. It can be seen that the estimation improves when SAILH and Kuusk CR models are used. For all indices used the estimations improve (linear regression slope progressively approaches unity) and RMSE significantly decreases when the optical indices are calculated using first $R_{\infty}$ and then CR models (Table I), although RMSE does not improve significantly when CR models are used. In addition, generally lower RMSE is found with $R_{\infty 2}$ and $R_{\infty 3}$ than with $R_{\text {SAILH }}$ and $R_{\text {Kuusk }}$. From the three infinite reflectance models used. $R_{\infty 3}$ (Hapke) and $R_{\infty 2}$ (Yamada and Fujimura) are the ones achieving best estimations, approaching the predictive ability of SAILH and Kuusk CR models.

\section{B. Estimation of $\mathrm{Chl}_{a+b}$ by Model Inversion}

SAILH and MCRM CR models and PROSPECT leaf model were used for $\mathrm{chl}_{a+b}$ estimation by inversion using 1998 and 
TABLE II

ESTIMATION OF $\mathrm{ch}_{a+b}$ OVER 12 ACER SACCHARUM M. STUDY SITES IN 1998 AND 1999 BY MODEL INVERSION AND SCALING-UP METHODS, WITH LEAF STRUCTURAL PARAMETER $N$ ESTIMATED BY INVERSION $(N=1.54,1998$; $N=1.43,1999)$. For SAILH AND PROSPECT MODEL INVERSION THREE

METHODS WERE USED: MiniMIZING A FUNCTION WITH ALL SPECTRAL ChanNELS Without WeIGHTING COEFFICIENTS $\left(w_{i}\right)$, WITH WEIGHTING COEFFICIENTS CALCULATED AS THE INVERSE OF THE REFLECTANCE $\left(w_{i}\right)$ AND BY A FUNCTION BASED ON THE OPTICAL INDEX $R_{750} / R_{710}$

\begin{tabular}{|c|c|c|c|c|c|}
\hline \multicolumn{3}{|c|}{ SAILH + PROSPECT } & $\begin{array}{c}\text { MCRM + PROSPECT } \\
\left(\mathbf{R}^{2}, \text { RMSE in } \mu g / \mathrm{cm}^{2}\right) \\
\end{array}$ & \multicolumn{2}{|c|}{$\begin{array}{c}\begin{array}{c}\text { SCALING-UP } \mathbf{R}_{75} / \mathbf{R}_{710} \\
\left(\mathbf{R}^{2}, \text { RMSE in } \mu \mathrm{g} / \mathrm{cm}^{2}\right)\end{array} \\
\end{array}$} \\
\hline 998 & 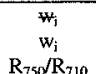 & $\begin{array}{l}0.33,12.0 \\
0.32,16.8 \\
0.43,5.57\end{array}$ & $\begin{array}{l}22 \\
\mathrm{er})\end{array}$ & $\begin{array}{c}\mathrm{CR}_{\text {SALH }} \\
\mathrm{R}_{\infty 03}\end{array}$ & \\
\hline 199 & $\begin{array}{c}w_{w_{i}} \\
w_{i} \\
R_{75} / R_{710}\end{array}$ & $\begin{array}{l}0.49,5.91 \\
0.47,11.43 \\
0.57,12.48\end{array}$ & $0.34,5.89$ & $\begin{array}{c}\mathrm{CR}_{\mathrm{SAIH} H} \\
\mathrm{R}_{\infty, 3}\end{array}$ & $\begin{array}{l}0.57,11.4 \\
0.57,8.3\end{array}$ \\
\hline
\end{tabular}

1999 CASI data. The inversion of the coupled MCRM and PROSPECT models was performed as indicated in [26] by minimizing a merit function $F[49]$. Two different approaches were tested: 1 ) setting $\mathrm{LAI}$ to a measured value $\mathrm{LAI}=4$; and 2) allowing both LAI and $\mathrm{chl}_{a+b}$ to vary. Other model parameters for MCRM were set to nominal values derived from the study areas, such as plagiophile LADF with $\theta_{m}=45^{\circ}, \epsilon=0.95$, solar zenith angle $\theta_{\mathrm{s}}=35^{\circ}$, hotspot parameter $s / l=0.008$, $N$ (PROSPECT) $=1.54$ (1998) and 1.41 (1999) estimated with collected leaf samples by PROSPECT model inversion, Markov parameter $\lambda_{\mathrm{z}}=1.1$, viewing angle $\theta=0^{\circ}$, relative sun azimuth $\psi=0^{\circ}$, and turbidity factor $\beta=0.18$.

The inversion of SAILH + PROSPECT models was performed by iteration and minimizing a function as indicated in [24], [25] for all the 72 CASI channels in the visible and NIR. In addition, a methodology consisting of minimizing a function based in a red edge optical index that show good correlation with pigment content in the leaf-level study was also used, where both $\mathrm{chl}_{a+b}$ and LAI were allowed to vary over a range of values four to seven for LAI and ten to $70 \mu \mathrm{g} / \mathrm{cm}^{2}$ for $\mathrm{chl}_{a+b}$, with the merit function adopted as in (4)

$$
\begin{aligned}
F\left(\mathrm{LAI}, \mathrm{chl}_{a+b}\right)=[ & \left(\frac{R_{750}}{R_{710}}\right)_{\mathrm{CASI}} \\
& \left.-\left(\frac{R_{750}}{R_{710}}\right)_{\mathrm{SAILH}+\text { PROSPECT }}\right]^{2} .
\end{aligned}
$$

A comparison of results from the two estimation methodologies is shown in Table II, with $R^{2}=0.33$ and RMSE = $12.0 \mu \mathrm{g} / \mathrm{cm}^{2}, R^{2}=0.49$ and RMSE $=5.91 \mu \mathrm{g} / \mathrm{cm}^{2}$ (1999) when all CASI channels were used as a minimizing function with no weighting coefficients $w_{i}$ and with LAI $=4$. When coefficients $w_{i}$ are specified as the inverse of the measured reflectance, results are $R^{2}=0.32$ and RMSE $=16.8 \mu \mathrm{g} / \mathrm{cm}^{2}$ (1998), and $R^{2}=0.47$ and RMSE $=11.43 \mu \mathrm{g} / \mathrm{cm}^{2}$ (1999), showing that RMSE increases when weighting coefficients are used.

Results obtained from the coupled MCRM and PROSPECT model inversion show $R^{2}=0.33$ and RMSE $=8.22 \mu \mathrm{g} / \mathrm{cm}^{2}$ (one outlier) in 1998; and $R^{2}=0.34$ and RMSE $=5.89 \mu \mathrm{g} / \mathrm{cm}^{2}$ in 1999 , therefore, indicating similar RMSE but lower $R^{2}$ compared to SAILH and PROSPECT inversion.

The results obtained with SAILH and PROSPECT model inversions using the red edge index $R_{750} / R_{710}$ as a min-
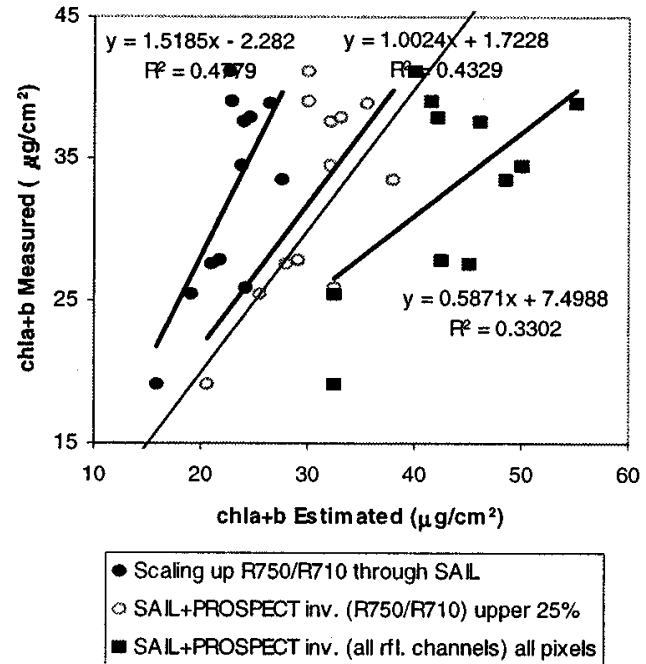

- SAlL+PROSPECT inv. (all $\mathrm{fl}$. channels) all pixels

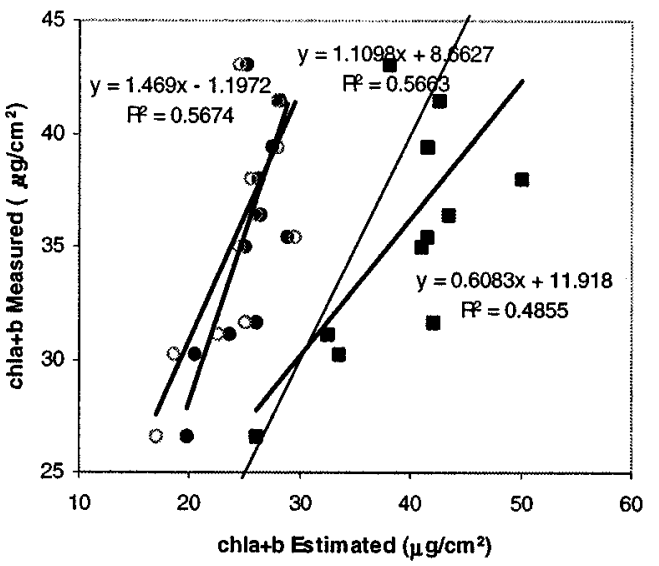

- Scaling up R750/R710 through SAl
o SAlL+PROSPECT inv. (R750/R710)
SAlL+PROSPECT inv. (all rtl. channels)

Fig. 5. Estimation of $\mathrm{chl}_{a+b}$ over 12 Acer saccharum M. study sites in 1998 (top) and 1999 (bottom) by 1) SAILH and PROSPECT model inversion using all reflectance channels in the merit function; 2) SAILH and PROSPECT model inversion using $R_{750} / R_{710}$ in the merit function; and 3) scaling-up $R_{750} / R_{710}$ through SAILH canopy reflectance model. LAI was set to four, and $N=1.54(1998), N=1.41(1999)$, estimated from leaf samples by PROSPECT model inversion.

imizing function by iteration are comparable to those from the scaling-up methodology, with $R^{2}=0.43$ and $\mathrm{RMSE}=5.57 \mu \mathrm{g} / \mathrm{cm}^{2} \mathrm{LAI}=4$ in 1998 and $R^{2}=0.57$ and RMSE $=12.48 \mu \mathrm{g} / \mathrm{cm}^{2}(\mathrm{LAI}=4)$ in 1999 . These results imply similar estimation performance as the scaling-up method for 1998 and 1999 using the same LAI: $R^{2}=0.47$ with RMSE $_{\text {SAILH }}=10.7 \mu \mathrm{g} / \mathrm{cm}^{2} ; \mathrm{RMSE}_{R_{\infty 3}}=8.11 \mu \mathrm{g} / \mathrm{cm}^{2}$ in 1998 and $R^{2}=0.57$, RMSE $E_{\text {SAILH }}=11.4 \mu \mathrm{g} / \mathrm{cm}^{2}$; RMSE $_{R_{\infty 3}}=8.3 \mu \mathrm{g} / \mathrm{cm}^{2}$ in 1999 (see Table II). Fig. 5 is used to illustrate the comparison of estimations for 1998 and 1999 by 1 ) scaling up $R_{750} / R_{710}$ index through SAILH; 2 ) by SAILH and PROSPECT inversion using all CASI reflectance channels; and 3) by SAILH + PROSPECT inversion using the red edge $R_{750} / R_{710}$ index.

Both the scaling-up and the SAILH + PROSPECT model inversion methods generated comparable results when the same 


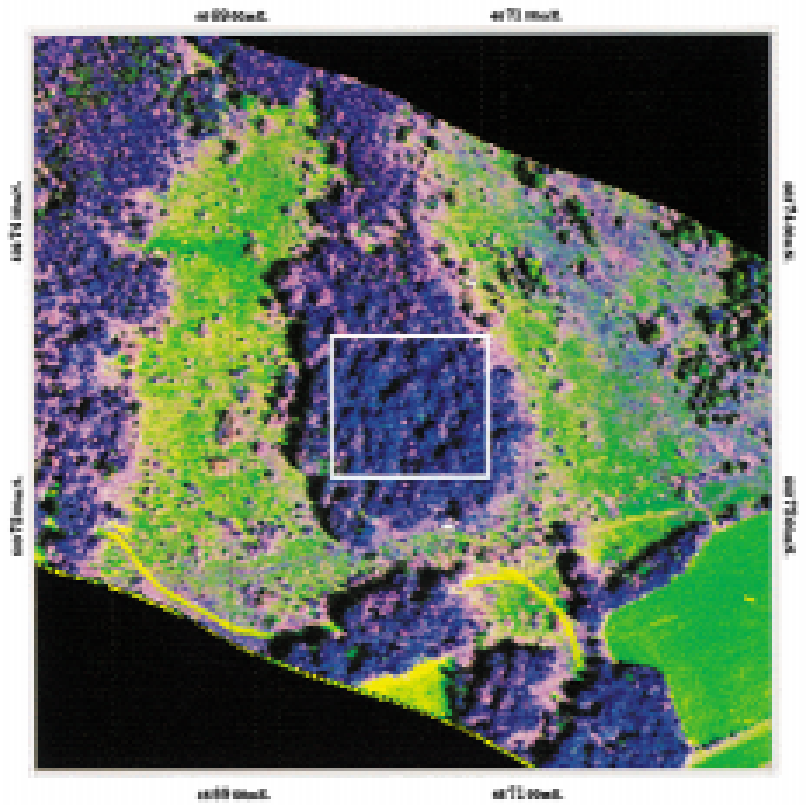

(a)

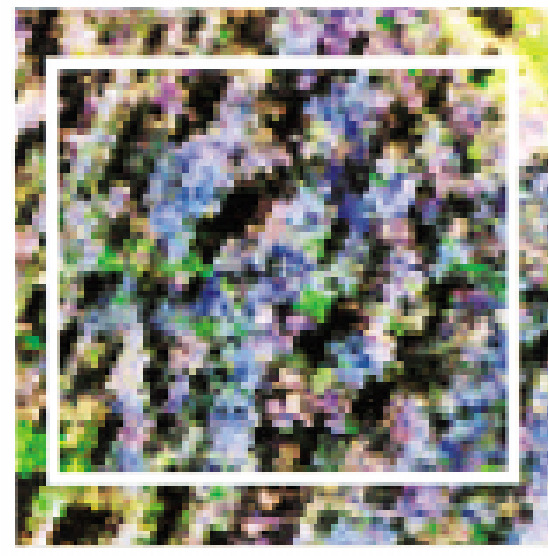

(b)

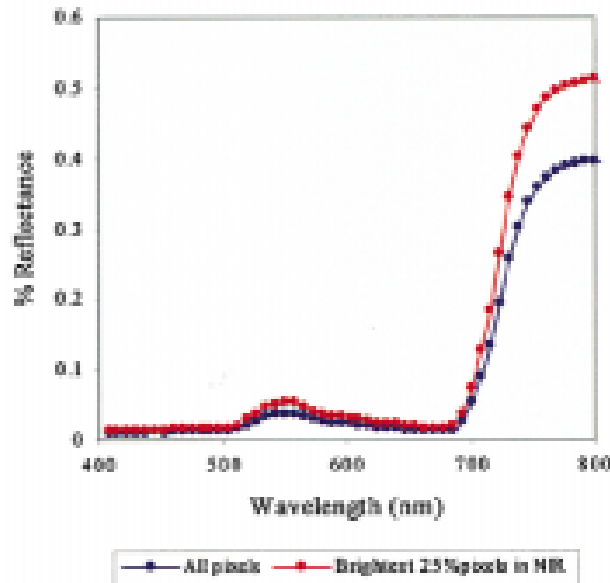

(d)

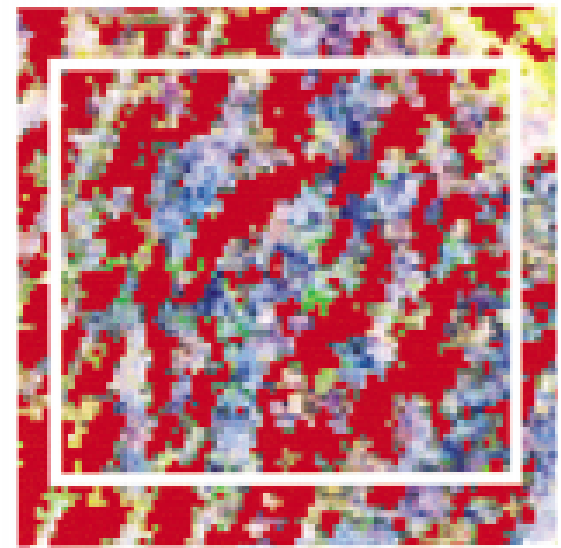

(c)

Fig. 6. Reflectance spectra obtained from one Acer saccharum M. study site by selection of all pixels (d, in blue) and targeting the brightest $25 \%$ in the NIR (d, in red). The plot (b)-(c) from the (a) study area is subset and a channel in the NIR ( $800 \mathrm{~nm})$ used to select the brightest pixels. Red pixels in (c) are those ones that are not selected when the spectrum is calculated targeting only the crowns, therefore not including shadows and canopy openings.

minimizing function based on a red edge index was used, and poorer estimations when all CASI reflectance bands are used. The small effect of LAI variation in closed dense canopies can be seen in the estimation of $\mathrm{chl}_{a+b}$ by SAILH + PROSPECT model inversion with all reflectance channels as merit function with $w_{i}=1 / r_{i}: R^{2}=0.32$, RMSE $=16.8 \mu \mathrm{g} / \mathrm{cm}^{2}$ for LAI $=$ 
4; $R^{2}=0.32, \mathrm{RMSE}=16.6 \mu \mathrm{g} / \mathrm{cm}^{2}$ for LAI variable (1998), and $R^{2}=0.47$, RMSE $=11.43 \mu \mathrm{g} / \mathrm{cm}^{2}$ for $\mathrm{LAI}=4 ; R^{2}=$ $0.41, \mathrm{RMSE}=12.16 \mu \mathrm{g} / \mathrm{cm}^{2}$ for LAI variable (1999). These results clearly demonstrate that minimizing functions based on absolute canopy reflectance bands, weighted or not as a function of wavelength, generate poorer determination coefficients and RMSEs than a single optical index calculated in the red edge spectral region, such as $R_{750} / R_{710}$.

\section{Effects of Shadows and Canopy Structure}

SAILH, Kuusk CR models and infinite reflectance models, used for model inversion or in the scaling-up method, provide an improvement in prediction capabilities compared to statistical leaf-level linear relationships. Nevertheless, both SAILH and Kuusk models are useful for infinite plane-parallel turbid-medium canopies, and the infinite reflectance models that correspond to a thick layer subject to the assumptions made for the multiple scattering. Canopy openings, shadows, and changes in the geometry of the canopy elements typical for broad leaf forest stands are not simulated in the modeling described above, therefore raising questions about the appropriateness of analysis using modeling approaches designed for uniform vegetation canopies. In order to use plane-parallel canopy model results with our CASI data, all the results discussed were obtained by a preselection of the upper pixels values in the NIR, therefore selecting the brightest pixels minimizing shadows and canopy openings in all study areas (Fig. 6). The different methodologies used here for pigment estimation were tested for both 1) calculation of the average reflectance from all $20 \times 20 \mathrm{~m}$ study sites by selection of the brightest $25 \%$ pixels in the NIR, as done before, and 2) calculation of the average of all pixels from the $20 \times 20 \mathrm{~m}$ sites, therefore including an effect due to shadows. Since we are dealing with a dense forest canopy and using high spatial resolution image data, the effects of the shadows on the different methodologies used for pigment estimation can be studied. The results for 1998 and 1999 CASI data are summarized in Tables III and IV, for eight methods of pigment estimation: 1,2 , and 3 scale up the $R_{750} / R_{710}$ optical index through $R_{\infty 1}, R_{\infty 2}$ and $R_{\infty 33}$ infinite reflectance models, respectively; 4 scales up the $R_{750} / R_{710}$ optical index through SAILH canopy reflectance model; 5 and 6 are numerical inversions of SAILH and PROSPECT using all reflectance channels in the merit function, and using $R_{750} / R_{710}$ in the merit function, respectively; and 7 and 8 are numerical inversions of $R_{\infty 2}$ and $R_{\infty 3}$, respectively, coupled with PROSPECT using $R_{750} / R_{710}$ in the merit function.

It is clear that there is little effect due to shadows in the estimation of the $\operatorname{chl}_{a+b}$ when the red edge optical index $R_{750} / R_{710}$ is used in the merit function for all the methods used: RMSE $=5.57 \mu \mathrm{g} / \mathrm{cm}^{2}$ (brightest $25 \%$ ), MSE $=5.48 \mu \mathrm{g} / \mathrm{cm}^{2}$ (all pixels) in 1998 with SAILH and PROSPECT inversion using $R_{750} / R_{710}$ in the merit function. On the other hand, a large effect is found due to radiometric texture (shadows) when all reflectance channels are used in the minimizing function: RMSE $=12 \mu \mathrm{g} / \mathrm{cm}^{2}$ (brightest $25 \%$ ), RMSE $=23.1 \mu \mathrm{g} / \mathrm{cm}^{2}$ (all pixels) in 1998 with SAILH and PROSPECT inversion. This finding is consistent in all cases (Tables III and IV), demonstrating that optical indices in the
TABLE III

COMPARISON OF RMSE $\left(\mu \mathrm{g} / \mathrm{cm}^{2}\right)$ AND $R^{2}$ FOR $\mathrm{ch}_{a+b}$ ESTIMATION BY SCALING-UP LEAF-LEVEL OPTICAL INDICES IN 1998 AND 1999 DEPLOYMENTS CONSIDERING All PiXels IN THE $20 \times 20 \mathrm{~m}$ AREA AVERAGEd REFLECTANCE WITH 2 M SPATIAL RESOlUtion AND 72-CHANNEL CASI DATA (100 PIXELS), AND Selecting THE UPPER 25\% PIXELS IN THE NIR TO Minimize SHADOWS AND OPENINGS IN A DENSE CANOPY OF ACER SACCHARUM M

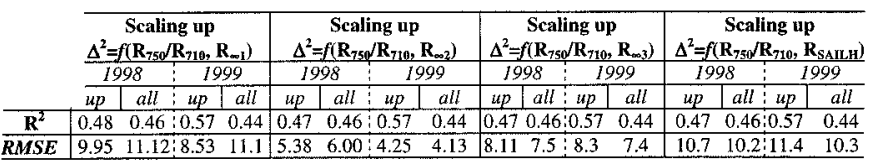

TABLE IV

COMPARISON OF RMSE $\left(\mu \mathrm{g} / \mathrm{cm}^{2}\right)$ AND $R^{2}$ FOR $\mathrm{ch} 1_{a+b}$ ESTIMATION IN 1998 AND 1999 DEPLOYMENTS CONSIDERING ALL PIXELS IN THE $20 \times 20 \mathrm{~m}$ AREA AVERAGED REFLECTANCE WITH 2 M SPATIAL RESOLUTION AND 72-CHANNEL CASI DATA (100 PiXelS), AND SELECTING THE UPPER 25\% PIXELS IN THE NIR to Minimize Shadows AND OpENINGS IN a DENSE CANOPY OF ACER SACCHARUM M. LAI $=4$ WAS CONSIDERED IN ALL CASES, AND NO WeIGHTING COEFFICIENTS WERE USED IN THE MODEL INVERSION WHEN ALL ChanNels ARE USED IN THE MERIT FunCtion

\begin{tabular}{|c|c|c|c|c|c|c|c|c|c|c|c|c|c|c|}
\hline & \multicolumn{4}{|c|}{$\begin{array}{c}\text { SAILH+PROSPECT } \\
\Delta^{2}=f\left(\Sigma \mathbf{R}_{\lambda}\right)\end{array}$} & \multicolumn{4}{|c|}{$\begin{array}{c}\text { SAILH+PROSPECT } \\
\Delta^{2}=f\left(\mathbf{R}_{750} / \mathbf{R}_{710}\right)\end{array}$} & \multicolumn{2}{|c|}{$\begin{array}{c}\mathbf{R}_{\infty 22}+\text { PROSPECT } \\
\Delta^{2}=f\left(\mathbf{R}_{750} / \mathbf{R}_{71, i}\right)\end{array}$} & \multicolumn{4}{|c|}{$\begin{array}{c}\mathbf{R}_{\infty 3}+\text { PROSPECT } \\
\Delta^{2}=f\left(\mathbf{R}_{750} / \mathbf{R}_{710}\right)\end{array}$} \\
\hline & & 98 & & 299 & 19 & 98 & 19 & 999 & 1998 & 1999 & & 98 & 19 & 999 \\
\hline & & ain & pe & all & $u p$ & all & & all & \begin{tabular}{l|l} 
up & all \\
up
\end{tabular} & al & $m$ & all & $u p$ & all \\
\hline $\mathbf{R}^{2}$ & 0 & 0.34 & 0.48 & 0.11 & 0.43 & 0.41 & 0.57 & 0.47 & $0.470 .45: 0.57$ & 70 & .47 & 0. & 0.56 & 0.45 \\
\hline $\boldsymbol{I S E}$ & & 23.1 & 5.9 & 16.7 & 5.57 & 5.48 & 12.48 & 11.01 & \begin{tabular}{ll|l}
22.5 & 24.2 & 8.9 \\
\end{tabular} & 1. & 5.36 & 6.03 & 35.13 & 4.35 \\
\hline
\end{tabular}

red edge are less affected to structural changes and shadows than single reflectance channels. This result is illustrated in Fig. 7, where it can be seen that red edge $R_{750} / R_{710}$ optical index used for model inversion through canopy modeling is not perceptibly affected when all pixels are included in the averaged reflectance from the $20 \times 20 \mathrm{~m}$ study sites $(2 \times 2 \mathrm{~m}$ pixel size), thereby including canopy shadows and openings.

This analysis also demonstrates that $\mathrm{chl}_{a+b}$ estimation by scaling up through $R_{\infty 2}$ infinite reflectance model is the methodology, which generates the smallest RMSE, although higher errors are found when the same infinite reflectance model is used coupled with PROSPECT and inverted. This suggests that leaf-level derived relationships are more accurate for scaling up through $R_{\infty 2}$ than reflectance and transmittance modeled by PROSPECT. If no leaf-level relationships are derived, model inversion using $R_{\infty 3}+$ PROSPECT showed lower RMSE compared to SAILH + PROSPECT, each with $R_{750} / R_{710}$ in the merit function. Estimation of $\mathrm{chl}_{a+b}$ over the study sites that presented extreme values of $\mathrm{chl}_{a+b}$ measured in the field in 1998 and 1999 campaigns are shown in Fig. 8, with the highest values measured in leaf samples of $38.8 \mu \mathrm{g} / \mathrm{cm}^{2}$ (1998, Fig. 8, upper left) and $45.8 \mu \mathrm{g} / \mathrm{cm}^{2}$ (1999, Fig. 8, lower left). The lowest values of $\mathrm{chl}_{a+b}$ measured were $19.08 \mu \mathrm{g} / \mathrm{cm}^{2}$ (1998, Fig. 8, upper right) and $26.58 \mu \mathrm{g} / \mathrm{cm}^{2}$ (1999, Fig. 8, lower right).

Three numerical model inversion methods that showed best results in 1998 and 1999 for $\mathrm{chl}_{a+b}$ estimation were used for the validation carried out in 2000 deployment with a different set of study sites: 1) SAILH coupled with PROSPECT with all 72 CASI channels in the minimizing merit function, with no weighting function; 2) SAILH coupled with PROSPECT ( $N=1.4$ ) with $R_{750} / R_{710}$ optical index as merit function; and 3) $R_{\infty 3}$ coupled with PROSPECT $\left(N=1.4\right.$ ) with $R_{750} / R_{710}$ optical index as merit function. The results obtained for the 


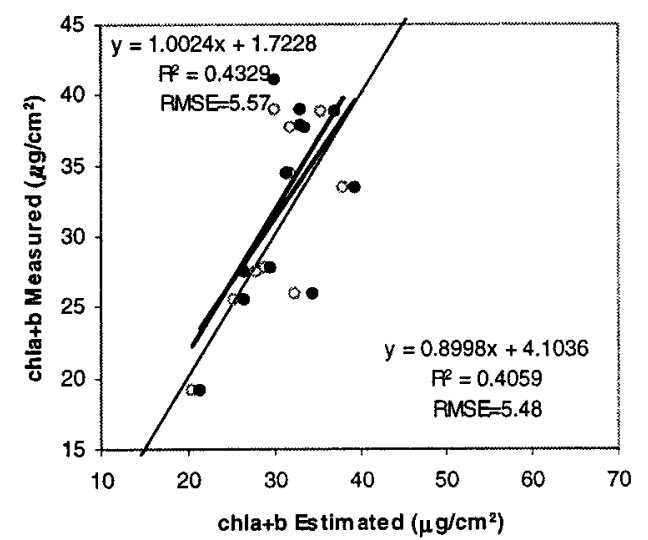

O SALL+PROSPECT inv. (R750/R710) upper $25 \%$

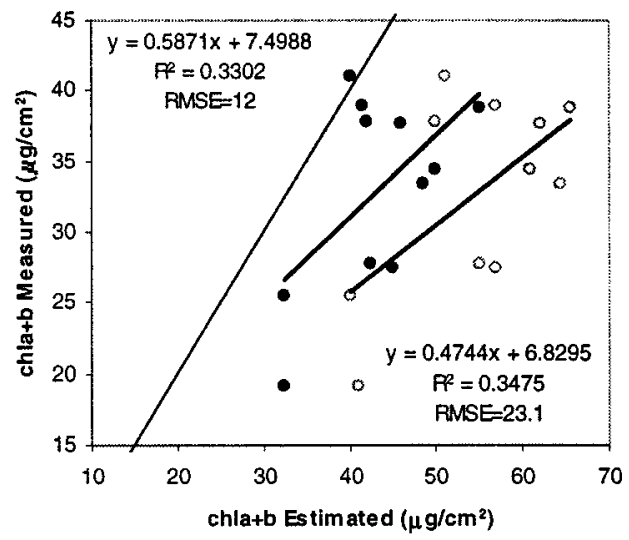

O SAIL+PROSPECT inv. (all ifl. channels) upper $25 \%$ - SAlL+PROSPECT inv. (all rfl. channets) all pixels

Fig. 7. Effect of shadows and canopy structure in the estimation of $c h 1_{a+b}$ by SAILH + PROSPECT model inversion using (left) $R_{750} / R_{710}$ and (right) all CASI spectral channels in the merit function. The red-edge $R_{750} / R_{710}$ optical index used for model inversion through canopy modeling does not get affected when all pixels are included in the averaged reflectance from the $20 \times 20 \mathrm{~m}$ study sites $(2 \times 2 \mathrm{~m}$ pixel size), therefore including canopy shadows (data from the 1998 campaign).
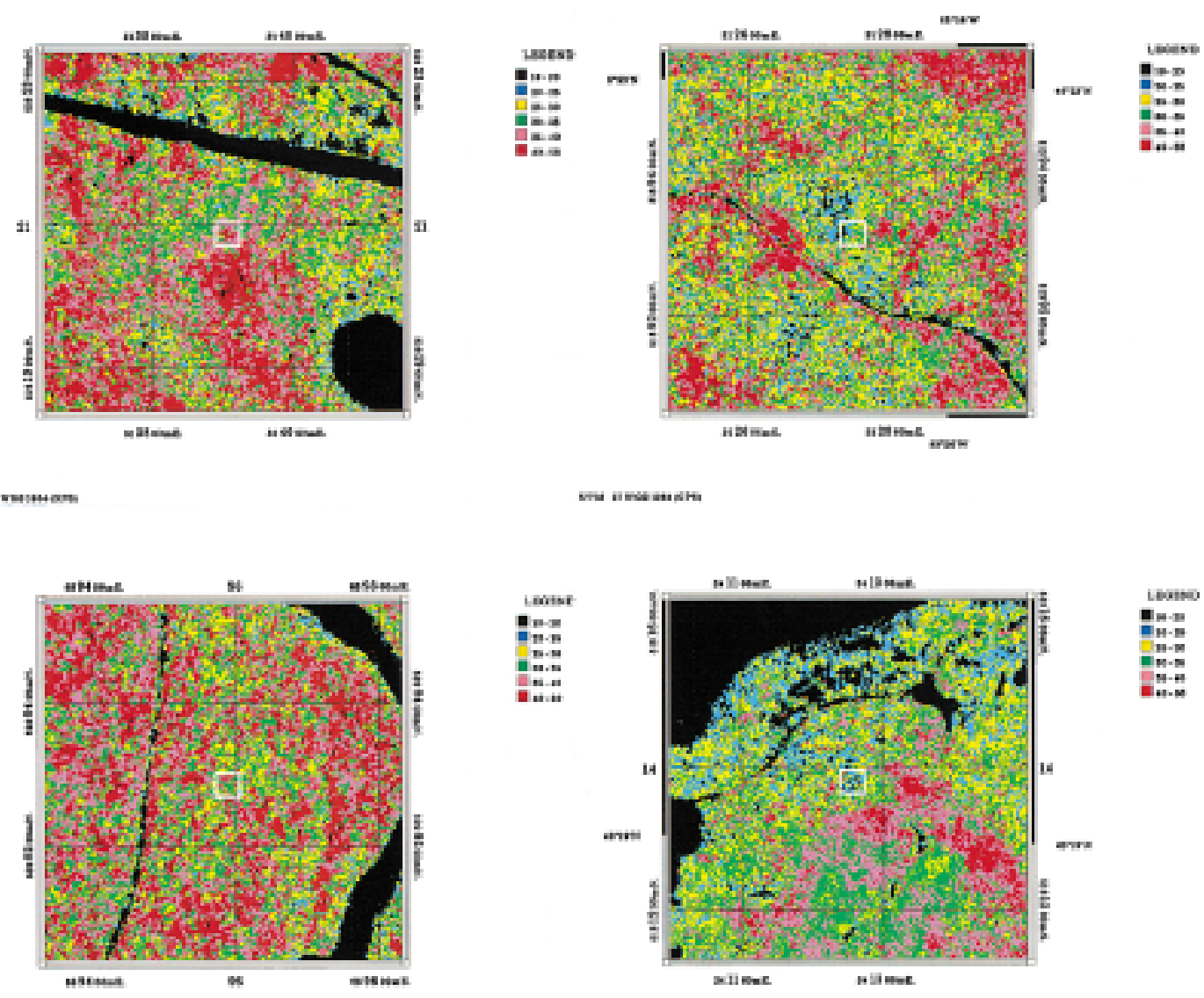

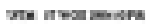

Fig. 8. Chl ${ }_{a+b}$ estimation over the study areas $(500 \times 500 \mathrm{~m})$ that presented extreme values of $\mathrm{chl}_{a+b}$ measured in the field in (up) 1998 and (bottom) 1999 campaigns. The highest values of $\mathrm{chl}_{a+b}$ (left) were measured in leaf samples from GY41 network site (upper left, $\mathrm{ch} 1_{a+b}$ measured $=38.8 \mu \mathrm{g} / \mathrm{cm}^{2}$ in 1998 ) and GY15 (lower left, $\mathrm{chl}_{a+b}$ measured $=45.8 \mu \mathrm{g} / \mathrm{cm}^{2}$ in 1999). The lowest values of (right) $\mathrm{chl}_{a+b}$ were measured in leaf samples from MD35 (upper right, $\mathrm{chl}_{a+b}$ measured $=19.08 \mu \mathrm{g} / \mathrm{cm}^{2}$ in 1998) and MD33 (lower left, $\mathrm{chl}_{a+b}$ measured $=26.58 \mu \mathrm{g} / \mathrm{cm}^{2}$ in 1999). White box shows the study area of $20 \times 20 \mathrm{~m}$ where leaf sampling was carried out.

three methods when all pixels and only the brightest $25 \%$ pixels in the NIR are targeted in the $80 \times 80 \mathrm{~m}$ plots are summarized in Table V. These results confirm the 1998 and
1999 conclusions, demonstrating that better estimations are achieved when the merit function is based on a red edge optical index such as $R_{750} / R_{710}: R^{2}=0.18$, RMSE $=14.8 \mu \mathrm{g} / \mathrm{cm}^{2}$ 


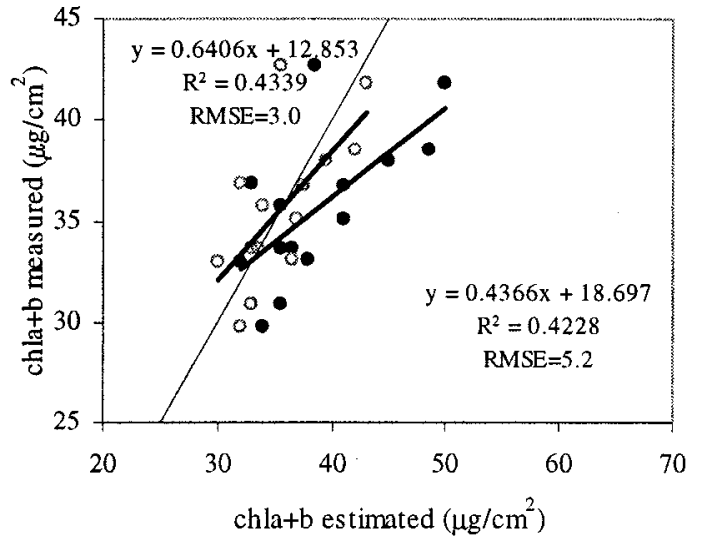

- SAIL+PROSPECT inv. (R750/R710), all pixels O SAIL+PROSPECT inv. (R750/R710), upper 25\%

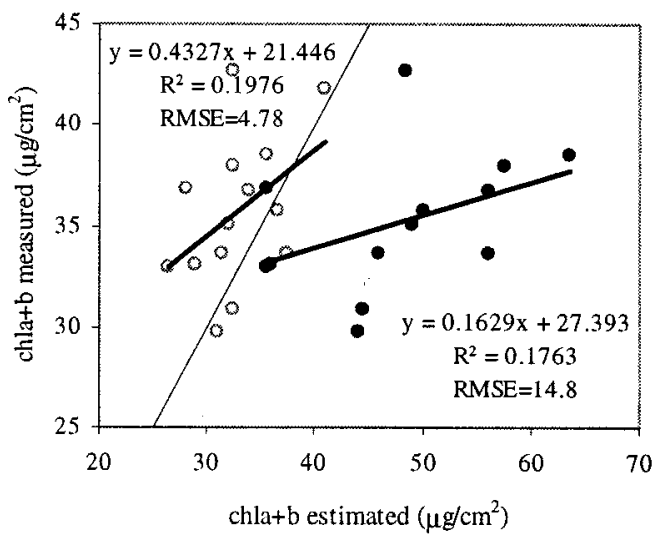

- SAIL+PROSPECT inv. (all rfl. channels), all pixels

$\because$ SAIL+PROSPECT inv. (all rfl. channels), upper $25 \%$

Fig. 9. Effect of shadows in the estimation of $\mathrm{ch} 1_{a+b}$ by SAILH + PROSPECT model inversion using (right) $R_{750} / R_{710}$ and (left) all CASI reflectance channels in the merit function confirmed with the CASI 2000 data. The red edge $R_{750} / R_{710}$ optical index used for model inversion through canopy modeling does not get as much as affected when all pixels are included in the averaged reflectance from the $80 \times 80 \mathrm{~m}$ study sites $(0.86 \times 3.4 \mathrm{~m}$ resampled to $1.5 \times 1.5 \mathrm{~m}$ pixel size), therefore including canopy shadows.

TABLE V

COMPARISON OF RMSE AND $R^{2}$ FOR $\mathrm{ch} 1_{a+b}$ ESTIMATION IN THE YEAR 2000 VALIDATION DEPLOYMENT CONSIDERING ALL PIXELS IN THE $80 \times 80 \mathrm{~m}$

AREA AVERAGED REFLECTANCE OF $0.86 \times 3.4 \mathrm{~m}$ RESAMPLED TO $1.5 \times 1.5 \mathrm{~m}$ SPATIAL RESOLUTION AND 72-CHANNEL CASI DATA (2809 PIXELS), AND SELECTING THE UPPER 25\% PIXELS IN THE NIR TO Minimize SHADOWS AND OPENINGS IN A DENSE CANOPY OF ACER SACCHARUM M. LAI $=4$ WAS CONSIDERED IN All CASES, AND No WeIGHTING COEFFICIENTS WERE USED IN THE MODEL INVERSION

\begin{tabular}{|c|c|c|c|c|c|c|}
\hline & \multicolumn{2}{|c|}{$\begin{array}{c}\text { SAILH+ } \\
\text { PROSPECT } \\
\Delta^{2}=f\left(\Sigma R_{\lambda}\right) \\
\end{array}$} & \multicolumn{2}{|c|}{$\begin{array}{c}\text { SAILH+ } \\
\text { PROSPECT } \\
\Delta^{2}=f\left(\mathbf{R}_{750} / \mathbf{R}_{710}\right) \\
\end{array}$} & \multicolumn{2}{|c|}{$\begin{array}{c}\mathbf{R}_{\infty 33}+ \\
\text { PROSPECT } \\
\Delta^{2}=f\left(\mathbf{R}_{750} / \mathbf{R}_{710}\right)\end{array}$} \\
\hline & up & all & $u p$ & all & $u p$ & all \\
\hline $\mathbf{R}^{2}$ & 0.2 & 0.18 & 0.42 & 0.43 & 0.43 & 0.44 \\
\hline RMSE & 4.8 & 14.8 & 3.0 & 5.2 & 5.0 & 8.9 \\
\hline
\end{tabular}

(SAILH + PROSPECT inversion using all reflectance channels, all pixels); $R^{2}=0.43$, RMSE $=5.2 \mu \mathrm{g} / \mathrm{cm}^{2}$ (SAILH + PROSPECT inversion using $R_{750} / R_{710}$ red edge index, all pixels).

The small effect of shadows on pigment estimation when $R_{750} / R_{710}$ index is used with SAILH + PROSPECT model inversion was also confirmed: RMSE $=3.0 \mu \mathrm{g} / \mathrm{cm}^{2}$ (brightest $25 \%$ ), $\mathrm{RMSE}=5.2 \mu \mathrm{g} / \mathrm{cm}^{2}$ (all pixels), and a larger variation in RMSE is observed when all reflectance channels are used: RMSE $=4.8 \mu \mathrm{g} / \mathrm{cm}^{2}$ (brightest $25 \%$ ), RMSE $=14.8 \mu \mathrm{g} / \mathrm{cm}^{2}$ (all pixels) (see Fig. 9). Results obtained with $R_{\infty 3}$ infinite reflectance model coupled with PROSPECT also demonstrate its applicability in closed canopies with high LAI. PROSPECT coupled with SAILH and $R_{\infty 3}$ with $R_{750} / R_{710}$ in the merit function obtained similar results $\left(R^{2}=0.4\right)$, although RMSE's are slightly smaller with SAILH: RMSE $=3.0 \mu \mathrm{g} / \mathrm{cm}^{2}$ (SAILH + PROSPECT, brightest $25 \%)$, RMSE $=5.0 \mu \mathrm{g} / \mathrm{cm}^{2}\left(R_{\infty 3}+\right.$ PROSPECT, brightest $25 \%) ;$ RMSE $=5.2 \mu \mathrm{g} / \mathrm{cm}^{2}$ (SAILH + PROSPECT, all pixels $),$ RMSE $=8.9 \mu \mathrm{g} / \mathrm{cm}^{2}\left(R_{\infty 3}+\right.$ PROSPECT, all pixels).

\section{CONCLUSIONS}

Radiative transfer theory and modeling assumptions were applied at leaf, laboratory, and field scales in order to study the link between leaf reflectance and transmittance and canopy airborne hyperspectral data acquired with different spectral and spatial characteristics. Approaches to $\mathrm{chl}_{a+b}$ estimation in closed forest canopies of Acer saccharum M. focused on both scaling-up and numerical model inversion approaches. A methodology for linking leaf-level relationships between optical indices and pigment content to canopy-level reflectance was presented. It has been demonstrated that leaf-level relationships calculated from single leaf reflectance and transmittance data collected from the ground can be scaled-up to above-canopy level through infinite reflectance and canopy reflectance models using nominal input parameters derived for these study areas consisting of closed canopies. The high spatial resolution of the airborne hyperspectral CASI data permitted the selection of crowns, eliminating shadows and understorey, therefore allowing the use of SAILH and Kuusk turbid-medium canopy models.

The results obtained in the scaling-up approach through canopy reflectance models and hyperspectral canopy reflectance from Acer saccharum M. study sites showed that red-edge indices, especially $\lambda_{\mathrm{p}}$ and DP21 $\left(D_{\lambda \mathrm{p}} / D_{703}\right)$, and spectral and derivative indices such as $R_{750} / R_{710}, \quad \operatorname{Vog} 1 \quad\left(R_{740} / R_{720}\right), \quad$ G_M2 $\left(R_{750} / R_{700}\right), \operatorname{Vog} 3\left(R_{734}-R_{747}\right) /\left(R_{715}+R_{720}\right), \operatorname{Vog} 2$ $\left(R_{734}-R_{747}\right) /\left(R_{715}+R_{726}\right), \operatorname{Vog} 4\left(D_{715} / D_{705}\right)$, G_M1 $\left(R_{750} / R_{550}\right)$, Ctr2 $\left(R_{695} / R_{760}\right)$ are the best optical indices for ch $1_{a+b}$ estimation at canopy level. Furthermore, traditional and widely used optical indices for pigment estimation and indicators of vegetation status, such as NDVI and SR, performed poorly in the two consecutive years. These traditional indices primarily track canopy structural changes but are not able to track subtle changes due to pigment content variation between 
study sites. This demonstrates that canopies with homogeneous structure but different chlorophyll content need the use of red edge and spectral indices to estimate changes in pigment content.

For the closed Acer saccharum M. canopies studied in this research, the $\mathrm{chl}_{a+b}$ estimations using optically thick models, which don't need structural and viewing geometry as input parameters implying much faster and easier operational applicability, demonstrated a predictive potential (low RSME in estimations) that was close to, and for some indices, superior to those using canopy models. Of the three infinite reflectance models used, the formulae $R_{\infty 3}$ (Hapke) and $R_{\infty 2}$ (Yamada and Fujimura) provided the best estimations, suggesting that infinite reflectance models can be used for canopy reflectance modeling in closed forest canopies of high LAI, performing as well as canopy reflectance models when crowns are targeted and specific sensitive indices are used.

The approach of scaling-up of optical indices through canopy models was compared to the numerical model inversion of coupled PROSPECT leaf radiative transfer model with SAILH, MCRM and infinite reflectance models, in which no leaf sampling is required to develop the statistical relationships. Results of the numerical model inversion by iteration showed that superior results were found when a methodology consisting on minimizing a function based in a red edge optical index was used, rather than by matching all the CASI reflectance bands in the visible and NIR. Results of SAILH and PROSPECT coupled model inversion using the red edge index $R_{750} / R_{710}$ as a minimizing function by iteration showed comparable results to the scaling-up methodology, without the need for developing leaf-level relationships. Furthermore, estimations show only a small effect when shadow pixels are included for the estimation of the $\operatorname{chl}_{a+b}$ using SAILH and PROSPECT inversion with the red edge optical index $R_{750} / R_{710}$ in the merit function. On the other hand, a large effect due to inclusion of shadow pixels is found with SAILH and PROSPECT inversion when all reflectance bands are used in the minimizing function. This result might have important implications, showing the value of red edge indices for both scaling-up and model inversion approaches in pigment estimation of closed canopies.

Results obtained in this research for three consecutive years, two of them with extensive leaf sampling campaigns, using a set of 12 Acer saccharum M. sites, were validated with a new set of 14 sites in order to test the conclusions. The successful validation campaign carried out in the summer of 2000 confirmed the hypothesis developed in previous years, obtaining comparable RMSE for $\mathrm{chl}_{a+b}$ estimation by numerical model inversion of coupled SAILH and PROSPECT models with nominal input parameters and $R_{750} / R_{710}$ in the merit function. Results presented in this paper demonstrate the capability of high spatial hyperspectral sensors to map pigment content over closed forest canopies through radiative transfer models. Moreover, it suggests that methodologies investigated here based on pigment estimation by model inversion using red edge indices might be transferred to MERIS sensor using the $R_{750} / R_{705}$ optical index, although the implications of the spatial heterogeneity of the forest landscape compared to the $300 \mathrm{~m}$ sensor spatial resolution, and the species dependence will need to be evaluated.

\section{ACKNOWLEDGMENT}

The authors wish to thank their colleagues who have contributed: J. Harron, L. Gray, J. Freemantle, P. Shepherd, P. Brasher, and H. Beck, whose dedication and skill made the airborne CASI field campaigns work. They also wish to thank D. Irving, B. Brown, N. Symour, M. Packalen, D. Hickie, and S. Martin, who planned and executed complex site sampling campaigns. Finally, they wish to thank N. Goel, A. Kuusk, W. Verhoef, and S. Jacquemoud for the provision of computer code for leaf and canopy reflectance models.

\section{REFERENCES}

[1] P. J. Curran, "Estimating Green LAI from Multispectral Aerial Photography," Photogramm. Eng. Remote Sensing, vol. 49, pp. 1709-1720, 1983.

[2] M. J. Farabee, "Photosynthesis," [Onlline] Avaialble: http://gened.emc.maricopa.edu/bio/bio181/BIOBK/BiobookPS.html, 1997.

[3] B. N. Rock, T. Hoshizaki, and J. R. Miller, "Comparison of In Situ and airborne spectral measurements of the blue shift associated with forest decline," Remote Sens. Environ., vol. 24, pp. 109-127, 1988.

[4] J. E. Vogelmann, B. N. Rock, and D. M. Moss, "Red edge spectral measurements from sugar maple leaves," Int. J. Remote Sensing, vol. 14, pp. $1563-1575,1993$

[5] G. A. Carter, "Ratios of leaf reflectances in narrow wavebands as indicators of plant stress," Int. J. Remote Sensing, vol. 15, pp. 697-704, 1994.

[6] A. A. Gitelson and M. N. Merzlyak, "Signature analysis of leaf reflectance spectra: Algorithm development for remote sensing of chlorophyll," J. Plant Physiol., vol. 148, pp. 494-500, 1996.

[7] P. J. Zarco-Tejada and J. R. Miller, "Land cover mapping at BOREAS using red edge spectral parameters from CASI imagery," J. Geophys. Res., vol. 104, pp. 27921-27933, 1999.

[8] P. J. Zarco-Tejada, J. R. Miller, G. H. Mohammed, T. L. Noland, and P. H. Sampson, "Canopy optical indices from infinite reflectance and canopy reflectance models for forest condition monitoring: Application to hyperspectral CASI data," in IEEE 1999 Int. Geoscience and Remote Sensing Symp., IGARSS'99, Hamburg, Germany, 1999.

[9] — - "Optical Indices as Bioindicators of Forest Condition from Hyperspectral CASI data," in Proc. 19th Symp. Eur. Assoc. Remote Sensing Laboratories (EARSeL), Valladolid, Spain, 1999.

[10] J. Peñuelas, I. Filella, J. Llusia, D. Siscart, and J. Piñol, "Comparative field study of spring and summer leaf gas exchange and photobiology of the Mediterranean trees Quercus ilex and Phillyrea latifolia," J. Exp. Bot., vol. 49, pp. 229-238, 1998.

[11] J. A. Gamon, L. Serrano, and J. S. Surfus, "The photochemical reflectance index: An optical indicator of photosynthetic radiation-use efficiency across species, functional types, and nutrient levels," Oecologia, vol. 112, pp. 492-501, 1997.

[12] J. Peñuelas, J. Llusia, J. Piñol, and I. Filella, "Photochemical reflectance index and leaf photosynthetic radiation-use-efficiency assessment in Mediterranean trees," Int. J. Remote Sensing, vol. 18, pp. 2863-2868, 1997.

[13] A. A. Gitelson, C. Buschman, and H. K. Lichtenthaler, "The chlorophyll fluorescence ratio F735/F700 as an accurate measure of chlorophyll content in plants," Remote Sens. Environ., vol. 69, pp. 296-302, 1999.

[14] J. A. Gamon and J. S. Surfus, "Assessing leaf pigment content and activity with a reflectometer," New. Phytol, vol. 143, pp. 105-117, 1999.

[15] P. J. Zarco-Tejada, J. R. Miller, G. H. Mohammed, and T. L. Noland, "Chlorophyll fluorescence effects on vegetation apparent reflectance: I. Leaf-level measurements and model simulation," Remote Sens. Environ., 2000.

[16] P. J. Zarco-Tejada, J. R. Miller, G. H. Mohammed, T. L. Noland, and P. H. Sampson, "Chlorophyll fluorescence effects on vegetation apparent reflectance: II. Laboratory and airborne canopy-level measurements with hyperspectral data," Remote Sens. Environ., to be published.

[17] P. J. Zarco-Tejada, "Hyperspectral remote sensing of closed forest canopies: Estimation of chlorophyll fluorescence and pigment content," Ph.D. dissertation, York Univ., Toronto, ON, Canada, 2000.

[18] J. R. Miller, E. W. Hare, and J. Wu, "Quantitative characterization of the vegetation red edge reflectance: an inverted-Gaussian model," Int. J. Remote Sensing, vol. 11, pp. 1755-1773, 1990. 
[19] L. F. Johnson, C. A. Hlavka, and D. L. Peterson, "Multivariate analysis of AVIRIS data for canopy biochemical estimation along the Oregon transect," Remote Sens. Environ., vol. 47, pp. 216-230, 1994.

[20] P. Matson, L. Johnson, C. Billow, J. Miller, and R. Pu, "Seasonal patterns and remote spectral estimation of canopy chemistry across the Oregon transect," Ecol. Applicat., vol. 4, pp. 280-298, 1994.

[21] D. L. Peterson, J. D. Aber, P. A. Matson, D. H. Card, N. Swanberg, C. Wessman, and M. Spanner, "Remote sensing of forest canopy and leaf biochemical contents," Remote Sens. Environ., vol. 24, pp. 85-108, 1988.

[22] B. J. Yoder and R. E. Pettigrew-Crosby, "Predicting nitrogen and chlorophyll content and concentrations from reflectance spectra (400-2500 $\mathrm{nm})$ at leaf and canopy scales," Remote Sens. Environ., vol. 53, pp. 199-211, 1995.

[23] F. Zagolski, V. Pinel, J. Romier, D. Alcayde, J. Fotanari, J. P. Gastellu-Etchegorry, G. Giordano, G. Marty, and R. Joffre, "Forest canopy chemistry with high spectral resolution remote sensing," Int. J. Remote Sensing, vol. 17, pp. 1107-1128, 1996.

[24] S. Jacquemoud, "Inversion of the PROSPECT + SAIL canopy reflectance model from AVIRIS equivalent spectra: theoretical study," Remote Sens. Environ., vol. 44, pp. 281-292, 1993.

[25] S. Jacquemoud, F. Baret, B. Andrieu, F. M. Danson, and K. Jaggard, "Extraction of vegetation biophysical parameters by inversion of the PROSPECT + SAIL models on sugar beet canopy reflectance data. Application to TM and AVIRIS sensors," Remote Sens. Environ., vol. 52, pp. 163-172, 1995.

[26] A. Kuusk, "Monitoring of vegetation parameters on large areas by the inversion of a canopy reflectance model," Int. J. Remote Sensing, vol. 19, pp. 2893-2905, 1998.

[27] V. Demarez and J. P. Gastellu-Etchegorry, "A modeling approach for studying forest chlorophyll content," Remote Sens. Environ., vol. 71, pp. 226-238, 2000

[28] M. Weiss, F. Baret, R. B. Myneni, A. Pragnere, and Y. Knyazikhin, "Investigation of a model inversion technique to estimate canopy biophysical variables from spectral and directional reflectance data," Agronomie, vol. 20, pp. 3-22, 2000.

[29] S. Jacquemoud, C. Bacour, H. Poilve, and J. P. Frangi, "Comparison of four radiative transfer models to simulate plant canopies reflectancedirect and inverse mode," Remote Sens. Environ., vol. 74, pp. 471-481, 2000.

[30] J. M. Chen and T. A. Black, "Defining leaf area index for nonflat leaves," Plant Cell Environ, vol. 15, pp. 421-429, 1992.

[31] J. M. Chen, P. M. Rich, S. T. Gower, J. M. Norman, and S. Plummer, "Leaf area index of boreal forests: Theory, techniques and measurements," J. Geophys. Res., vol. 102, no. D24, pp. 29429-29 443.

[32] E. W. Chappelle, M. S. Kim, and J. E. I. McMurtrey, "Ratio analysis of reflectance spectra (RARS): an algorithm for the remote estimation of the concentrations of chlorophyll $a$, chlorophyll $b$, and carotenoids in soybean leaves," Remote Sens. Environ., vol. 39, pp. 239-247, 1992.

[33] A. A. Gitelson and M. N. Merzlyak, "Remote estimation of chlorophyll content in higher plant leaves," Int. J. Remote Sensing, vol. 18, pp. 2691-2697, 1997.

[34] J. A. Kupiec and P. J. Curran, "Decoupling effects of the canopy and foliar biochemicals in AVIRIS spectra," Int. J. Remote Sensing, vol. 16, pp. 1731-1739, 1995.

[35] A. Pragnère, F. Baret, M. Weiss, R. B. Myneni, Y. Knyazikhin, and L. B. Wang, "Comparison of three radiative transfer model inversion techniques to estimate canopy biophysical variables from remote sensing data," in Proc. Int. Geoscience and Remote Sensing Symp. (IGARSS'99), Hamburg, Germany, 1999.

[36] B. H. Braswell, D. S. Schimel, J. L. Privette, Moore III, W. J. Emery, E. W. Sulzman, and A. T. Hudak, "Extracting ecological and biophysical information from AVHRR optical data: An integrated algorithm based on inverse modeling," J. Geophys. Res., vol. 101, no. D18, pp. 23 335-23 348, 1996

[37] M. Weiss and F. Baret, "Evaluation of canopy biophysical variable retrieval performances from the accumulation of large swath satellite data," Remote Sens. Environ., vol. 70, pp. 293-306, 1999.

[38] W. Verhoef, "Light scattering by leaf layers with application to canopy reflectance modeling: the SAIL model," Remote Sens. Environ., vol. 16, pp. $125-141,1984$.

[39] A. Kuusk, "A Markov chain model of canopy reflectance," Agr. Forest Meteorol., vol. 76, pp. 221-236, 1995.

[40] —-, "A computer-efficient plant canopy reflectance model," Comput. Geosci., vol. 22, pp. 149-163, 1996
[41] O. Lillestaeter, "Spectral reflectance of partly transmitting leaves: Laboratory measurements and mathematical modeling," Remote Sens. Environ, vol. 12, pp. 247-254, 1982.

[42] J. R. Miller, M. D. Steven, and T. H. Demetriades-Shah, "Reflection of layered bean leaves over different soil backgrounds: measured and simulated spectra," Int. J. Remote Sensing, vol. 13, pp. 3273-3286, 1992.

[43] N. Yamada and S. Fujimura, "Nondestructive measurement of chlorophyll pigment content in plant leaves from three-color reflectance and transmittance," Appl. Opt., vol. 30, pp. 3964-3973, 1991.

[44] B. Hapke, Theory of Reflectance and Emittance Spectroscopy. Cambridge, U.K.: Univ. Press, 1993.

[45] P. Bicheron, M. Leroy, and O. Hautecoeur, "LAI and fAPAR mapping at global scale by model inversion against spaceborne POLDER data," in Proc. Int. Geoscience and Remote Sensing Symp. '98, Seattle, WA, 1998.

[46] N. S. Goel, "Models of vegetation canopy reflectance and their use in estimation of biophysical parameters from reflectance data," Remote Sensing Rev., vol. 4, pp. 1-212, 1988.

[47] A. A. Abuelgasim, S. Gopal, and A. H. Strahler, "Forward and inverse modeling of canopy directional reflectance using a neural network," Int. J. Remote Sensing, vol. 19, pp. 453-471, 1998.

[48] J. A. Smith, "LAI inversion using back propagation neural network trained with multiple scattering model," IEEE Trans. Geosci. Remote Sensing, vol. 31, pp. 1102-1106, 1993

[49] A. Kuusk, "Determination of vegetation canopy parameters from optical measurements," Remote Sens. Environ., vol. 37, pp. 207-218, 1991

[50] S. W. Running, C. O. Justice, V. Solomonson, D. Hall, J. Barker, Y. J. Kanfmann, A. H. Strahler, A. R. Huete, J. P. Muller, V. Vanderbilt, Z. M. Wan, P. Teilletland, and D. Carneggie, "Terrestrial remote sensing and algorithms planned for EOS/MODIS," Int. J. Remote Sensing, vol. 15, no. 17 , pp. $3587-3620,1994$.

[51] A. Huete and C. Justice, "MODIS Vegetation Index (MOD 13) Algorithm Theoretical Basis Document," Greenbelt: NASA Goddard Space Flight Centre, http://modarch.gsfc.nasa.gov/MODIS/LAND/\#vegetation-indices, 1999.

[52] N. T. O'Neill, F. Zagolski, M. Bergeron, A. Royer, J. R. Miller, and J. Freemantle, "Atmospheric correction validation of casi images acquired over the BOREAS southern study area," Can. J. Remote Sensing, vol. 23, pp. $143-162,1997$.

[53] Anon, "A master plan to examine forest growth and dynamics in Ontario," Ont. Mining Nat. Res., p. 68, 1993.

[54] J. Hayden, J. Kerley, D. Carr, T. Kenedi, and J. Hallarn, "Field manual for establishing and measuring permanent sample plots," Ont. Mining Nat. Res., p. 161, 1995

[55] D. L. McLaughlin, D. E. Corrigan, and W. D. McIlveen, "Etiology of sugar maple decline at selected sites in Ontario (1984-1990)," Ontario Ministry Environ., ARB-052-92-PHYTO, 1992.

[56] D. L. McLaughlin, J. C. Kinch, H. Liljalehto, and E. Boysen, "Hardwood forest health surveys in Ontario-the first 10 years. Ontario Ministry Environment and Energy," Ontario Ministry Nat. Res., 1999.

[57] J. W. Harron and J. R. Miller, "An alternate methodology for reflectance and transmittance measurements of conifer needles," in 17th Can. Symp. Remote Sensing, vol. II, Saskatoon, SK, Canada, 1995, pp. 654-661.

[58] 1800-12 Integrating Sphere Instruction Manual, Li-Cor-Inc, 1983.

Pablo J. Zarco-Tejada received the degree in agricultural engineering from the School of Agriculture, Engineering and Forestry (ETSIAM), Universidad de Cordoba, Cordoba, Spain, the M.Sc. in remote sensing from the Department of Applied Physics and Electronics and Mechanical Engineering (APEME), University of Dundee, Dundee, U.K., and the Ph.D. in Eearth and space science from York University, Toronto, ON, Canada, in 2000.

His research is focused on hyperspectral remote sensing of vegetation, working with airborne sensors to estimate leaf biochemical and canopy biophysical parameters through inversion of leaf and canopy radiative transfer models, and optical indices. His recent work is related to modeling the chlorophyll fluorescence effects of leaf and canopy apparent reflectance through radiative transfer simulation. He is now a Postdoctoral Fellow with the Center for Spatial Technologies and Remote Sensing (CSTARS), University of California, Davis (UCD), working with MQDIS and ASTER sensors. He is continuing his involvement in hyperspectral research using Hyperion (EQ-i), AVIRJS, and CASL data over forest and agricultural canopies for pigment and water content estimation with specific interests in applications of hyperspectrai methodologies for precision agriculture. 


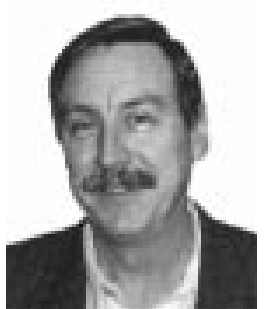

John R. Miller received the B.E. degree in physics and both the M.S.c. and Ph.D. degrees in space physics in 1963, 1966, and 1969, respectively, from the University of Saskatchewan, Saskatoon, SK, Canada, studying the aurora borealis using rocketborne radiometers.

He then spent two years on a Postdoctoral Fellowship at the Herzherg Institute at the National Research Council, Ottawa, ON, Canada. He joined the Faculty of York University, Toronto, ON, in 1972, where he is currently Professor of Physics and Director of the Earth Observations Laboratory of the Centre for Research in Earth and Space Technology (CRESTech). His remote sensing interests include atmospheric correction and extraction of biophysieal surface parameters through models from water color reflectance and forest canopy reflectance. Over the past decade, his primary focus has been on the application of reflectance spectroscopic techniques in remote sensing using imaging spectrometer sensors.

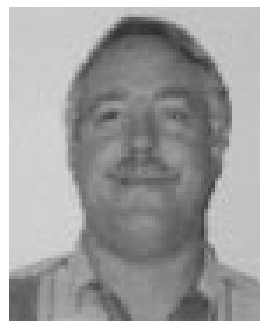

Thomas L. Noland received the B.S. degree in forest science from The Ohio State University, Columbus, in 1974, the M.S. in forestry from the University of Wisconsin, Madison, in 1977, and the Ph.D. in plant sciences from the University of Arkansas, Little Rock, in 1984, where his Ph.D. research was on the physiology and biochemistry of seed dormancy in sugar pine.

After three postdoctoral positions working on tree tissue culture, seed physiology, and plant molecular biology, he accepted the position as Tree Biochemist with the Ontario Forest Research Institute. His research interests are stress biochemistry, pigment biochemistry, and seed physiology and biochemistry. His current research projects range from the application of remote sensing in forestry to the effect of ice storm damage on maple sap and syrup production.
Gina H. Mohammed received the B.Sc. in biology from the University of Toronto, Toronto, ON, Canada, in 1981, and the Ph.D. in biology from Simon Fraser University, Burnaby, BC, Canada, in 1990.

She is currently Research Director of P\&M Technologies, Sault Ste. Marie, ON, Canada. Previously, she was a Research Scientist with the Ontario Ministry of Natural Resources. Her research background includes various aspects of plant science, including structure and function, detection and improvement of stress resistance, biotechnology, and nontimber forest products.

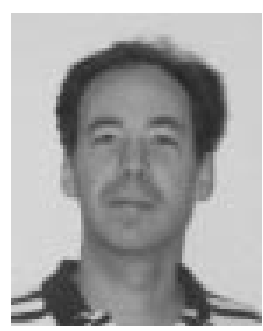

Paul H. Sampson received the B.Sc. degree in forestry from the University of Toronto, Toronto, ON, Canada, in 1989, and the M.Sc. degree in geography from York University, Toronto, in 2000.

$\mathrm{He}$ is a registered professional Forester, and his research interests include remote sensing, plant physiology, and forest ecology. 